\title{
Monin-Obukhov similarity theory and its application to wind flow modelling over complex terrain
}

\author{
Hendri J. Breedt ${ }^{\mathrm{a}}$, Ken J. Craig ${ }^{\mathrm{a}}$, Venkatesh D. Jothiprakasam ${ }^{\mathrm{b}}$ \\ ${ }^{a}$ Department of Mechanical and Aeronautical Engineering \\ University of Pretoria, Lynnwood Rd, Hatfield 0002, Pretoria, South Africa \\ ${ }^{b}$ Siemens Gamesa Renewable Energy, Borupvej, Brande 7330, Denmark
}

\begin{abstract}
In the wind resource industry Computational Fluid Dynamics (CFD) has gained widespread use to model the Atmospheric Boundary Layer (ABL). These models primarily focus on the neutrally stratified surface layer and ignore physical process such as buoyancy and the Coriolis force. Reductions in uncertainties of turbine suitability and energy production can be achieved if these processes are included. The present work focuses on the development and validation of an ABL CFD model using Monin-Obukhov Similarity Theory (MOST) in which atmospheric stability and the Coriolis force are included. MOST is applied to measured time series data obtained from a commercially proposed wind farm to determine the prevalence and impact of atmospheric stability. The analyses provide the inputs for the CFD model. The CFD model uses the standard $k-\epsilon$ turbulence model. To account for atmospheric stability modifications based on MOST are introduced to the standard CFD model equations. Two MOST models modifications are investigated. The modifications are successfully validated using the empty domain horizontal homogeneity test of the inlet profiles. The model is thereafter applied to the complex terrain of the proposed wind farm. The models are successfully validated by cross-prediction of the stability-dependent wind velocity profiles between two onsite meteorological masts.
\end{abstract}

Keywords: Atmospheric Boundary Layer, Atmospheric Stability, Monin-Obukhov Similarity Theory, Computational Fluid Dynamics, Buoyancy, $k-\epsilon$ turbulence model 


\section{Introduction}

In the wind resource assessment industry knowledge about the flow properties of the atmospheric boundary layer (ABL) is important, as the wind field is crucial to the design, suitability and performance of wind turbines. The wind fields vary spatially due to topographical influences and ground roughness changes. This results in significant variations in wind speed, wind direction and turbulence intensity across a wind farm. Site-specific information about the wind fields is obtained using on-site meteorological measurement masts or synthesized mesoscale data sets. Wind flow modelling is then used to extrapolate this information to areas on-site where no data are available. Computational Fluid Dynamics (CFD) is widely used for this application and focuses primarily on modelling the neutrally stratified atmospheric surface-layer. In order to reduce uncertainty in wind resource calculations it is necessary to model the whole ABL and its physical mechanisms.

The ABL can be in three main states namely stable, neutral and unstable. In stable conditions ambient turbulence and vertical fluxes are suppressed by buoyancy forces. This suppression of turbulence leads to delays in wind turbine wake recovery and can lead to increased energy losses. The lack of vertical motion increases vertical wind shear and can lead to uneven wind turbine blade loading. Unstable conditions are characterized by higher ambient turbulence as well as an increased boundary layer height due to the vertical motion experienced. This increases turbulence affects the turbine blade fatigue loads. In a diurnal cycle stable conditions are typically seen at night with cooler land temperatures while unstable conditions appear in day times with elevated temperatures. Typically, non-neutral conditions dominate for mean wind speeds lower than $15 \mathrm{~m} \mathrm{~s}^{-1}$ [1] [2]. Non-neutral conditions are thus present during the most prevalent wind speed conditions experienced by commercial wind farms.

This work focuses on the development of a CFD model for the ABL using a Reynolds-Averaged Navier-Stokes (RANS) model. The model must be able to solve both neutral and non-neutral flow over a typical complex wind farm terrain with its parameters derived from onsite time series wind data. MoninObukhov Similarity Theory (MOST) is applied to measured time series data from meteorological masts at the proposed wind farm location to understand the effects of atmospheric stability and to obtain the characteristic values. Modifications based on MOST are made to the standard RANS CFD model equations to account for atmospheric stability. These modifications are tested 
to be in equilibrium using an empty domain horizontal homogeneity test. Two $k-\epsilon$ turbulence model modifications for MOST are investigated: the methods of Alinot and Masson [3] and the recently proposed model from M.P. van der Laan et al. [1]. The models are applied to the wind farm location, to understand the model accuracy in complex terrain. The model is validated by cross predicting the velocity profiles obtained from the two meteorological masts.

In Section 2 a description of MOST and the ABL are given along with the corresponding assumptions. Section 3 is devoted to a review of the site location and the wind data analysis. Section 4 contains the computational settings and parameters of the CFD simulations, the model validation and results are presented in Section 5, followed by a discussion in Section 6 . finally, Section 7 concludes the work presented.

\section{Atmospheric boundary layer}

A full description and assumptions of the ABL and MOST can be found in the literature, e.g, Wallace and Hobbs [4] and Panofsky and Dutton [5]. For completeness of the current work a brief overview is presented here.

Considering only a neutrally stratified ABL the wind speed and temperature profiles are assumed to be accurately approximated with a logarithmic profile that reduces to zero at ground level. However, when stability is taken into account, the profile can deviate significantly from the standard neutral condition logarithmic profile [1] [4].

The Monin-Obukhov Length (MOL) is used to define atmospheric stability, as given in Equation 1, where $L$ denotes MOL, $u_{*}$ the frictional velocity, $T_{0}$ the ground surface temperature, $K$ the Von Karman constant and $\theta_{*}$ the temperature scale. The stability is defined in five classes as reported in Table 1 [6]. Up to seven classes exist including slightly unstable and slightly stable, for this work these cases are absorbed into the unstable and stable regions, respectively.

$$
L=\frac{u_{*}^{2} T_{0}}{K g \theta_{*}}
$$


Table 1: Monin-Obukhov Length classification for atmospheric stability [6]

\begin{tabular}{lr}
\hline Condition & Monin-Obukhov Length $[\mathrm{m}]$ \\
\hline Extremely Unstable & $-100 \leq L<0$ \\
Unstable & $-500 \leq L<-100$ \\
Neutral & $|L|>500$ \\
Stable & $50 \leq L<500$ \\
Extremely Stable & $0 \leq L<50$ \\
\hline
\end{tabular}

A conversion is made from temperature to potential temperature $\theta$ using Equation 2 [4], where $R$ is the universal gas constant, $p$ pressure, $p_{0}$ standard pressure at the earth's surface and $C_{p}$ specific heat. Taking the derivative of $\theta$ with respect to $\mathrm{z}$ (Height above ground) allows for the stability conditions to be easily recognized using Equation 3 [7].

$$
\begin{gathered}
\theta=T\left(\frac{p_{0}}{p}\right)^{R / C_{p}} \\
\frac{\partial \theta}{\partial z}>0 \quad \text { Unstable, } \quad \frac{\partial \theta}{\partial z}=0 \quad \text { Neutral, } \frac{\partial \theta}{\partial z}<0 \quad \text { Stable }
\end{gathered}
$$

Taking thermal stratification into account and converting to potential temperature, the wind speed and potential temperature profiles become

$$
\begin{aligned}
& u(z)=\frac{u_{*}}{K}\left[\ln \left(\frac{z}{z_{0}}\right)-\Psi_{m}\left(\frac{z}{L}\right)\right] \\
& \theta(z)=\theta\left(z_{0}\right)+\frac{\theta_{*}}{K}\left[\ln \left(\frac{z}{z_{0}}\right)-\Psi_{t}\left(\frac{z}{L}\right)\right]
\end{aligned}
$$

where $\Psi_{m}$ and $\Psi_{t}$ are the universal functions for wind speed and temperature based on the Dyer relations [8] given by Equations 6 to 11, where $z_{0}$ is the ground roughness length. For the neutral condition these universal functions are zero and recover the standard profiles. The profiles are functions of MOL, the calculation of MOL is not straightforward and various methods are presented in literature [9]. The method used in this work is based on a non-linear least squares fitting of Equations 4 and 5 to the multiple measurement heights on the onsite mast.

Stable:

$$
\Psi_{m}\left(\frac{z}{L}\right)=\Psi_{t}\left(\frac{z}{L}\right)=\frac{-5 z}{L}
$$


Neutral:

$$
\Psi_{m}\left(\frac{z}{L}\right)=\Psi_{t}\left(\frac{z}{L}\right)=0
$$

Unstable:

$$
\begin{gathered}
\Psi_{m}\left(\frac{z}{L}\right)=2 \ln \left[\frac{1+x}{2}\right]+\ln \left[\frac{1+x^{2}}{2}\right]-2 \arctan (x)+\frac{\pi}{2} \\
\Psi_{t}\left(\frac{z}{L}\right)=2 \ln \left[\frac{1+x^{2}}{2}\right]
\end{gathered}
$$

with

$$
x=\left[1-\left(16 \frac{z}{L}\right)\right]^{1 / 4}
$$

When modelling the ABL at full scale the Coriolis force due to the earth's rotation must be included [7]. The force is introduced into the RANS momentum equations using a source term $S_{M}$ as defined in Equation 12. $f_{c}$ is defined as the Coriolis parameter using Equation 13 with the earth's rotation rate $\Theta_{E}$ and latitude $\Lambda$ in geographical radians. The earth's rotation rate equals $7.292 \times 10^{-5} \mathrm{rad} \mathrm{s}^{-1}$ [4]. $U_{i}$ is the RANS velocity component along the respective Cartesian axis. The Coriolis force causes the air to deflect from its original path of motion and causes increasing wind veer as a function of height. Only the horizontal components are considered as the vertical component is negligible due to the gravitational acceleration.

$$
\begin{gathered}
S_{M}=\iota_{i} f_{c} \rho U_{i} \\
f_{c}=2 \Theta_{E} \sin (\Lambda) \quad, \quad \iota_{i}^{T}=(-1,1,0)
\end{gathered}
$$

\subsection{Turbulence modelling}

The wind flows considered in this work are classified as high Reynolds number flows and are based on numerical solutions of the RANS equations. The standard two-equation closure method for turbulence based on the $k-\epsilon$ turbulence model is utilized. The model uses the kinematic turbulent eddy viscosity in Equation 14. The model is based on two transport equations for turbulent kinetic energy $(k)$ and its dissipation rate $(\epsilon)$, as shown respectively in Equations 15 and 16 [10]. The model is presented in the form it appears in ANSYS Fluent 18.1 [10].

$$
v_{t}=C_{\mu} \frac{k^{3 / 4}}{\epsilon}
$$




$$
\begin{aligned}
& \frac{\partial \rho k}{\partial t}+\frac{\partial \rho k u_{i}}{\partial x_{i}}=\frac{\partial}{\partial x_{j}}\left[\left(\mu+\frac{\mu_{t}}{\sigma_{k}}\right) \frac{\partial k}{\partial x_{j}}\right]+G_{k}+G_{b}-\rho \epsilon-Y_{m}+S_{k} \\
& \frac{\partial \rho \epsilon}{\partial t}+\frac{\partial \rho \epsilon u_{i}}{\partial x_{i}}=\frac{\partial}{\partial x_{j}}\left[\left(\mu+\frac{\mu_{t}}{\sigma_{\epsilon}}\right) \frac{\partial \epsilon}{\partial x_{j}}\right]+C_{\epsilon 1} \frac{\epsilon}{k}\left(G_{k}+C_{\epsilon 3} G_{b}\right)-C_{\epsilon 2} \rho \frac{\epsilon^{2}}{k}+S_{\epsilon}
\end{aligned}
$$

$\sigma_{k}$ and $\sigma_{\epsilon}$ are the turbulent Prandtl numbers for $k$ and $\epsilon$ respectively with $S_{k}$ and $S_{\epsilon}$ user-defined source terms. $\rho$ is the air density and $C_{\epsilon 1}$ and $C_{\epsilon 2}$ are model constants. $G_{k}$ and $G_{b}$ represent turbulence production due to the mean velocity gradients and buoyancy respectively [10]. The degree to which $\epsilon$ is influenced by $G_{b}$ is determined by the $C_{\epsilon 3}$ constant.

\subsection{Monin-Obukhov similarity theory applied to the $k-\epsilon$ turbulence model}

Turbulence profiles generated using MOST are unbalanced with the standard $k-\epsilon$ transport equations [1] [3]. To accurately represent non-neutral conditions, modifications are required [3] [7]. In this work the methods of Alinot and Masson [3] and the recently proposed model from M.P. van der Laan et al. [1] are investigated, the models are hereafter referred to as the AM and DTU models respectively. Full details on the models can be found in their respective works. The AM model introduces $C_{\epsilon 3}$ as a function of the stability parameter $z / L$. The $\epsilon$ inlet profile is also modified to account for the $k$-equation imbalance. This method has been shown to work well for small domains [1] [3] however it can face issues in large domains due to the fact that the transport equation for $k$ is still not in equilibrium with MOST. The DTU model uses an additional analytical source term in the $k$-equation and a variable $C_{\epsilon 3}$. As a result the two equations of the model is in analytical balance with MOST [1]. Table 2 indicates the model constants $C_{\epsilon}$, Prandtl numbers $\sigma$ and MOST source terms $S_{k M O}$ required for the AM and DTU models as well as the standard model from Launder and Spalding (LS) [11] and the neutral model from Sorensen [12].

Table 2: Model constants for various $k-\epsilon$ models for ABL flows [1] [3] [11]

\begin{tabular}{lccccccccc}
\hline$k-\epsilon$ Model & $C_{\mu}$ & $K$ & $C_{\epsilon 1}$ & $C_{\epsilon 2}$ & $C_{\epsilon 3}$ & $\sigma_{k}$ & $\sigma_{\epsilon}$ & $\sigma_{\theta}$ & $S_{k M O}$ \\
\hline LS & 0.09 & 0.4 & 1.44 & 1.92 & 0 & 1 & 1.3 & 0.71 & - \\
Sorensen & 0.03 & 0.4 & 1.21 & 1.92 & 0 & 1 & 1.3 & - & - \\
AM & 0.033 & 0.42 & 1.176 & 1.92 & eq. 22 & 1 & 1.3 & 1 & - \\
DTU & 0.03 & 0.4 & 1.21 & 1.92 & eq. 27 & 1 & 1.3 & 1 & eq. 23 \\
\hline
\end{tabular}


Following the requirement that the transport equations must be in balance with the formulae used to specify the boundary conditions [13] and substituting the neutral profiles from Equations 4 and 5, one obtains the neutral turbulence profile Equations 17 and 18 [1].

$$
\begin{gathered}
k=\frac{u_{*}^{2}}{\sqrt{C_{\mu}}} \\
\epsilon(z)=\frac{u_{*}^{3}}{K z}
\end{gathered}
$$

A similar approach can be followed for the non-neutral boundary conditions following MOST for $k$ [1] and using measurements of the surface turbulent kinetic energy budget terms for $\epsilon$ [3]. This results in

$$
\begin{aligned}
& k(z)=\frac{u_{*}^{2}}{\sqrt{C_{\mu}}}\left(\frac{\psi_{\epsilon}}{\psi_{m}}\right)^{1 / 2} \\
& \epsilon(z)=\frac{u_{*}^{3}}{K z} \psi_{\epsilon}\left(\frac{z}{L}\right)
\end{aligned}
$$

where $\psi_{\epsilon}$ is based on the relation of Panofsky and Dutton [5]

$$
\psi_{\epsilon}= \begin{cases}1-\frac{z}{L} & , L<0 \\ \psi_{m}-\frac{z}{L} & , L>0\end{cases}
$$

and $\psi_{m}$ is the derivative of $\Psi_{m}$ with respect to $z$.

\subsubsection{Model I: $A M$}

The AM model uses the constants in Table 2 and $C_{\epsilon 3}$ is obtained using a fifth-order polynomial:

$$
C_{\epsilon 3}\left(\frac{z}{L}\right)=\sum_{n=0}^{5} a_{n}\left(\frac{z}{L}\right)^{n}
$$

with the coefficients listed in Table 3. The polynomial in Equation 22 is not a complete analytical solution but instead an approximation and is only valid for $-2.3<z / L<2.0$ [1]. 
Table 3: Alinot and Masson (AM) $C_{\epsilon 3}$ model constants [3]

\begin{tabular}{ccc|cc}
\hline & \multicolumn{2}{c|}{$L>0$} & \multicolumn{2}{c}{$L<0$} \\
& $\left(\frac{z}{L}\right)<0.33$ & $\left(\frac{z}{L}\right)>0.33$ & $\left(\frac{z}{L}\right)<-0.25$ & $\left(\frac{z}{L}\right)>-0.25$ \\
\hline$a_{0}$ & 4.181 & 5.225 & -0.0609 & 1.765 \\
$a_{1}$ & 33.994 & -5.269 & -33.672 & 17.1346 \\
$a_{2}$ & -442.398 & 5.115 & -546.88 & 19.165 \\
$a_{3}$ & 2368.12 & -2.406 & -3234.06 & 11.912 \\
$a_{4}$ & -6043.544 & 0.435 & -9490.792 & 3.821 \\
$a_{5}$ & 5970.776 & 0 & -11163.202 & 0.492 \\
\hline
\end{tabular}

\subsubsection{Model II: DTU}

The DTU method involves an additional source $S_{k M O}$ in the $k$-equation [1].

$$
S_{k M O}=\frac{u_{*}^{3}}{k z} \times \begin{cases}\left(\frac{L}{z}\right)\left(\psi_{m}-\psi_{\epsilon}\right)-\frac{\psi_{h}}{\sigma_{\theta} \psi_{m}}-\frac{C_{k D}}{4} \psi_{m}^{13 / 2} \psi_{\epsilon}^{-3 / 2} f_{u s}\left(\frac{z}{L}\right) & , L<0 \\ 1-\frac{\psi_{h}}{\sigma_{\theta} \psi_{m}}-\frac{C_{k D}}{4} \psi_{m}^{-7 / 2} \psi_{\epsilon}^{-3 / 2} f_{s t}\left(\frac{z}{L}\right) & , L>0\end{cases}
$$

employing the following stability functions:

$$
\begin{gathered}
C_{k D}=\frac{K^{2}}{\sigma_{k} \sqrt{C_{\mu}}} \\
f_{u s}\left(\frac{z}{L}\right)=\left(2-\frac{z}{L}\right)+\frac{16}{2}\left(1-12 \frac{z}{L}+7\left(\frac{z}{L}\right)^{2}\right)-16\left(3-54 \frac{z}{L}+35\left(\frac{z}{L}\right)^{2}\right) \\
f_{s t}\left(\frac{z}{L}\right)=\left(2-\frac{z}{L}\right)-10 \frac{z}{L}\left(1-2 \frac{z}{L}+10 \frac{z}{L}\right)
\end{gathered}
$$

Finally $C_{\epsilon 3}$ is determined using Equation 27.

$$
C_{\epsilon 3}=\frac{\sigma_{\theta} L}{z} \frac{\psi_{m}}{\psi_{h}}\left(C_{\epsilon 1} \psi_{m}-C_{\epsilon 2} \psi_{\epsilon}+\left[C_{\epsilon 2}-C_{\epsilon 1}\right] \psi_{\epsilon}^{-1 / 2} f_{\epsilon}\left(\frac{z}{L}\right)\right)
$$

with

$$
f_{\epsilon}\left(\frac{z}{L}\right)= \begin{cases}\psi_{m}^{5 / 2}\left(1-12 \frac{z}{L}\right) & , L<0 \\ \psi_{m}^{-5 / 2}\left(2 \psi_{m}-1\right) & , L>0\end{cases}
$$


For the DTU model $S_{k M O}$ and $C_{\epsilon 3}$ are complete analytical solutions to MOST and is valid for the entire range of $z / L[1]$ unlike the AM model.

Following the MOST assumptions, the standard $G_{b}$ formulation can be rewritten to yield Equation 29, shown here in its potential temperature form [1]. As before $\psi_{t}$ is the derivative of $\Psi_{t}$ with respect to $z$. This expression for $G_{b}$ is commonly used in literature [14] [15]. It can be considered as the ABL modeller's choice because it does not require a temperature gradient which allows MOST to be used without solving the energy equation and removes the issue where accurate steady simulations are difficult to obtain when buoyancy forces are present [1] [16]. In this study the MOST formulation is utilized.

$$
G_{b M O}=-\frac{g v_{t}}{\theta_{0} \sigma_{\theta}} \frac{\partial \theta}{\partial z}=-v_{t}\left(\frac{\partial U}{\partial z}\right)^{2} \frac{z \psi_{t}}{L \sigma_{\theta} \psi_{m}^{2}}
$$

\section{Data acquisition and analysis}

\subsection{Site description}

MOST as described in Section 2 is applied to measured time series data from onsite meteorological masts located on a proposed wind farm location in South Africa. The site is the study area utilized in this study and is characterized by a hill, running East-West, at $950 \mathrm{~m}$ above sea level (ASL) that drops down to $550 \mathrm{~m}$ ASL via steep and undulating terrain of up to $70^{\circ}$ of inclination. Surrounding the hill the site is predominately open and flat. Two meteorological masts are located on the hill approximately $7200 \mathrm{~m}$ apart. A graphical overview of the study area can be seen in Figure 1 with both mast locations shown, the pink and black spheres respectively shows the locations of Mast 1 and 2. Mast 1 is the primary mast and used for the current data analysis study. The ground cover is typical open farmland with no major obstacles, this corresponds to a roughness height $z_{0}$ of $0.030 \mathrm{~m}$ [17]. The digital terrain model of the site is constructed from surveyed $5 \mathrm{~m}$ contour data over and around the main hill and then extended with $30 \mathrm{~m}$ shuttle radar topography mission data [18] to obtain a site model of $35 \mathrm{~km} \times$ $25 \mathrm{~km}$. The $\mathrm{x}$ and $\mathrm{y}$ axes are aligned with East and North respectively. The study area is a commercial project and not an academic field experiment, therefor possible errors can be induced from the setup. However, the setup is accepted in the wind resource resource industry and follows all industry standards for a data measurement campaign [17]. 


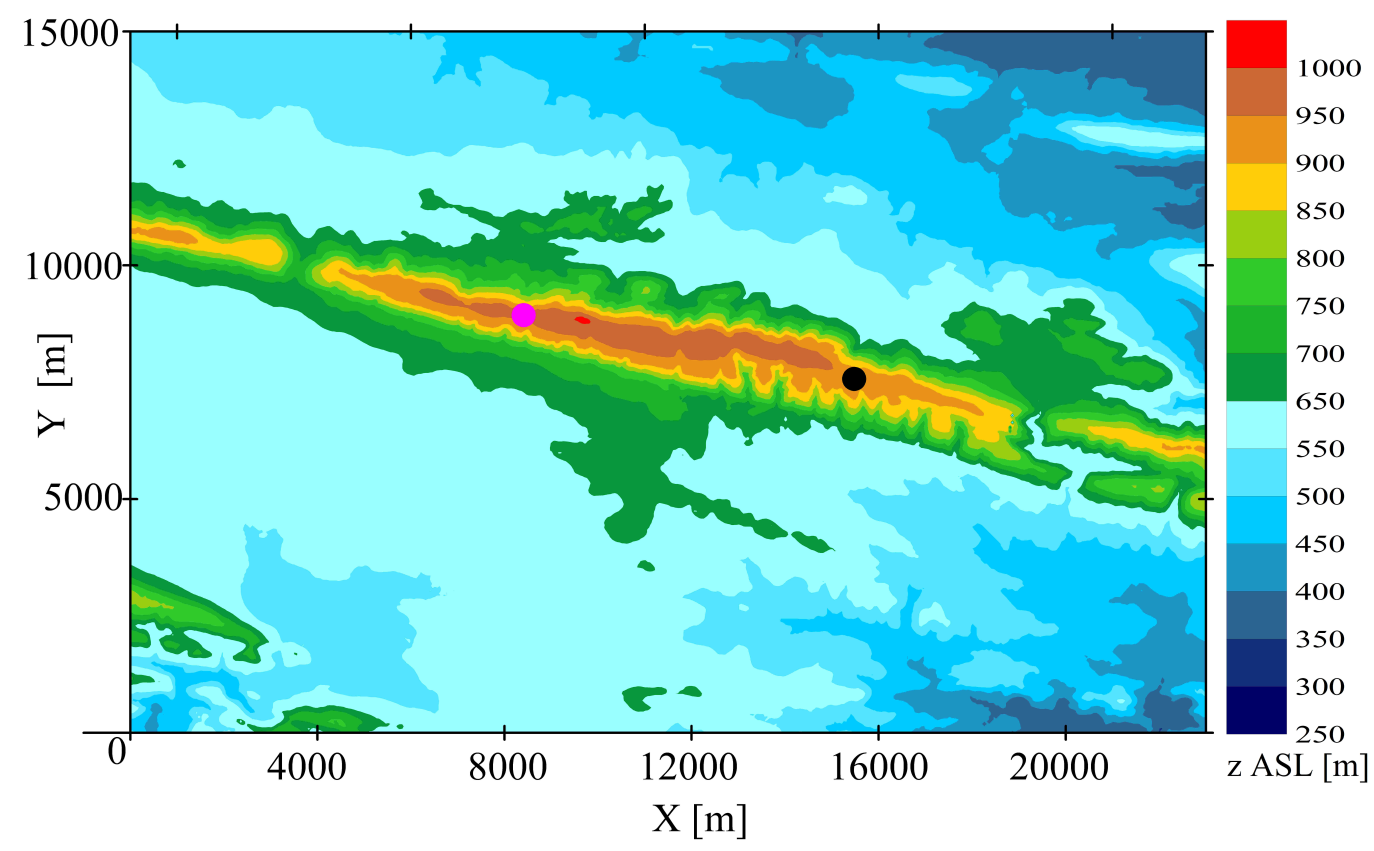

Figure 1: Overview of the study area

The masts used in this study are both $82 \mathrm{~m}$ tall with cup-anemometers located at $82 \mathrm{~m}, 60 \mathrm{~m}$, and $40 \mathrm{~m}$. Wind vanes are installed at $80 \mathrm{~m}$ and $40 \mathrm{~m}$ with temperature sensors at $80 \mathrm{~m}$ and $5 \mathrm{~m}$. Pressure and relative humidity are measured at $5 \mathrm{~m}$. Measnet Sensor calibration [19] has been successfully completed on all anemometers and wind vanes and the mast construction conforms to current commercial standards for mast installations. The terrain effects of the hill are expected to increase the wind z-velocity component. This results in a $\mathrm{y}-\mathrm{z}$ inflow angle at measurement heights. Well calibrated cup-anemometers such as those used in the study are however able to capture this change in inflow angle. Based on the Tilt Response Measurements from the CLASSCUP study [20] the cup-anemometers were able to accurately capture the effect from $0-10^{\circ}$ with negligible error. The anemometers measure mean and standard deviation. WRF (Weather Research and Forecasting) model data from EMD [21] were also downloaded at the same location. The model is a mesoscale numerical weather prediction system designed for both atmospheric research and operational forecasting and generates atmospheric simulations based on real data obtained from observations and analyses. Two full years of data (2015-2017) are utilised in this study, with the measured data and WRF data having resolutions of 10 minutes and 1 hour, respectively. 


\subsection{Prevalence of atmospheric stability}

To determine the frequency of each stability condition, the temperature was converted to potential temperature using Equation 2 for each reading. Using the potential temperature gradient and the conditions in Equation 3, the reading is classified as neutral, stable or unstable. The Monin-Obukhov Length of each reading is calculated using the three measurement heights for velocity to perform a non-linear least squares fit with the corresponding stability velocity profile from Equation 4 . Using the conditions in Table 1 the data are then binned into the various classes. The results for the measured and WRF data are shown in Table 4. The measured results show that only $11 \%$ is spent in the neutral condition, this shows that using the standard ABL CFD model for this site would be applicable to a very small portion of the actual onsite conditions. Comparing the measured and WRF data there is a negligible difference, expect for the unstable and neutral conditions, which can attributed to the fact that these conditions are non-dominating and statistically larger variations are present. In the two dominating conditions (extremely unstable and stable) only a 1\% difference is present, this shows the mesoscale data are able to capture stability for the site location.

Table 4: Stability classification difference between measured and mesoscale data

\begin{tabular}{cccccc}
\hline & $\begin{array}{c}\text { Extremely } \\
\text { Unstable }\end{array}$ & Unstable & Neutral & Stable & $\begin{array}{c}\text { Extremely } \\
\text { Stable }\end{array}$ \\
\hline Mast [\%] & 36 & 6 & 11 & 40 & 7 \\
WRF [\%] & 37 & 13 & 3 & 39 & 8 \\
\hline
\end{tabular}

The data were split into $12 \times 30^{\circ}$ bins using the top wind vane, the stability rose in Figure 2 shows the sector-wise distribution of stability from the measured data. The prevailing wind directions can be identified as sectors $120-180^{\circ}$. For this study sector $180^{\circ}$ (wind direction from $165-195^{\circ}$ ) is used as the test sector as it is one of the prevailing wind directions as well as being located directly south of the main hill with a long upwind fetch. 


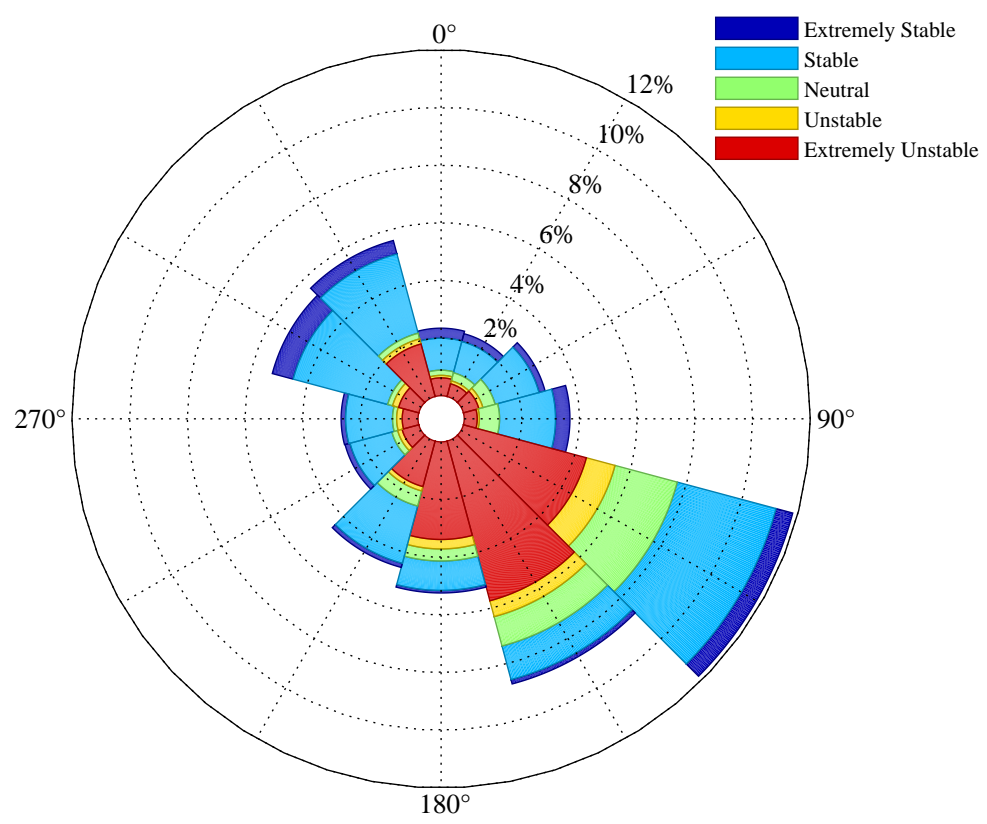

Figure 2: Stability rose for Mast 1

The 10 minute diurnal evolution of stability can be seen in Figure 3 based on the measured data. The main trend is identifiable with strong (90\%) extremely unstable and unstable prevalence during daytime with stable conditions dominating at night time. This diurnal cycle is used to average the data for all of the following calculations and any mean determined is weighted against the time it occurs during the diurnal cycle. For example, when determining statistics for the extremely stable region, the effects of the conditions occurring in night time is weighted more heavily than the few times it occurs during day time. This is done to alleviate the effects of stratification occurring outside of its normal conditions. 


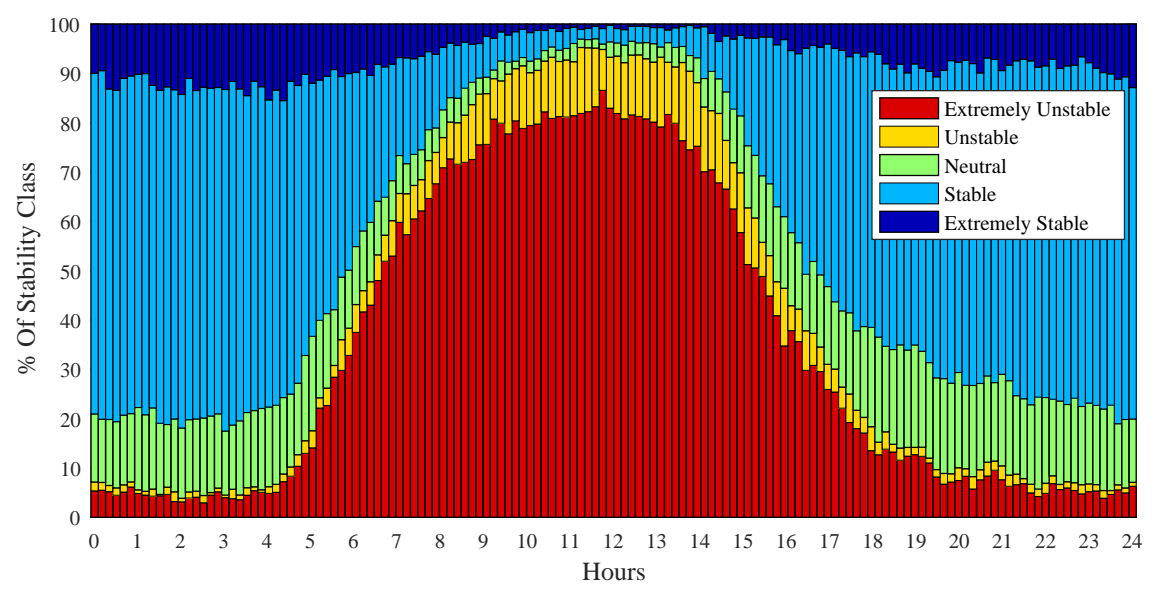

Figure 3: Diurnal stability classification for Mast 1

Three of the main conditions effecting turbine power performance and suitability are: wind speed, turbulence intensity and wind shear. Turbulence intensity is determined from anemometer data using the fraction of standard deviation to mean wind speed [17]. Wind shear is defined in terms of a shear exponent $\alpha$ as shown in Equation 30 using a power law for wind speed as a function of height $u(z)$ based on a reference wind speed at a fixed height $u\left(z_{r e f}\right)$. A larger shear exponent results in a greater increase in wind speed with height than a lower shear exponent. This equation is solved for $\alpha$ using a least-squares fit with data from the three measurement heights. This was completed for every reading to obtain the instantaneous shear exponent.

$$
\frac{u(z)}{u\left(z_{r e f}\right)}=\left(\frac{z}{z_{r e f}}\right)^{\alpha}
$$

The diurnal conditions are analyzed by assuming the central limit theorem allowing the mean to be taken at each 10 minute bin of the measured data by fitting a normal distribution at each time step. This results in the mean 24 hour profile of the measured conditions, the resulting profiles for turbulence intensity and shear exponent are shown in Figure 4. In the extremely unstable condition the turbulence intensity is much higher than in any other condition. The shear results indicate that in the extremely unstable and unstable region the shear exponent is very low due to the vertical motion of the air that limits wind profile growth, while the extremely stable and stable conditions both show very high wind shear values as expected. 

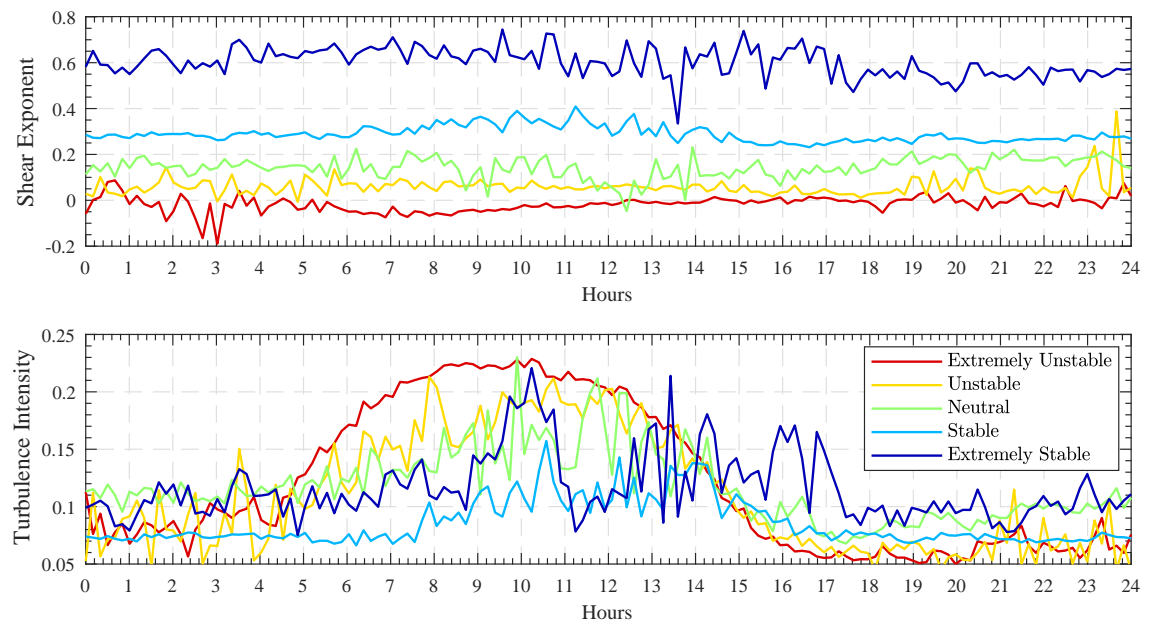

Figure 4: Diurnal turbulence intensity and wind shear exponent

Based on the stability prevalence results it is clear that non-neutral stratification is present on the site and that it influences the conditions to such an extent that using only the standard neutral CFD model the necessary effects would not be captured onsite.

\subsection{Vertical profiles}

Profiles for velocity, temperature and turbulence are calculated using the measured data from sector $180^{\circ}$. Using the diurnally weighted average of the data at each height a fixed data point for velocity, potential temperature and MOL is calculated for each stability condition. This process yields the results in Table 5.

Table 5: Average measured velocity, potential temperature and MOL - Sector $180^{\circ}$

\begin{tabular}{cccccc}
\hline & $\begin{array}{c}\text { Extremely } \\
\text { Unstable }\end{array}$ & Unstable & Neutral & Stable & $\begin{array}{c}\text { Extremely } \\
\text { Stable }\end{array}$ \\
\hline$u_{82}\left[\mathrm{~ms}^{-1}\right]$ & 7.00 & 8.25 & 8.10 & 5.68 & 2.65 \\
$u_{60}\left[\mathrm{~ms}^{-1}\right]$ & 6.95 & 8.13 & 7.90 & 5.29 & 2.19 \\
$u_{40}\left[\mathrm{~ms}^{-1}\right]$ & 6.97 & 7.86 & 7.71 & 4.87 & 1.76 \\
$\theta_{80}[\mathrm{~K}]$ & 299.6 & 299.4 & 294.1 & 295.3 & 294.1 \\
$\theta_{5}[\mathrm{~K}]$ & 298.4 & 298.5 & 294.2 & 296.0 & 294.8 \\
MOL $[\mathrm{m}]$ & -5.8 & -230.0 & N/A & 221.8 & 26.3 \\
\hline
\end{tabular}


Using Equation 4 with the corresponding stability functions in Equation 6 to 11 and the data from Table 5 in a non-linear least squares fit results in the solution of the frictional velocity $u_{*}$ to be obtained such that the velocity profile is the best fit to the data. The results for frictional velocity are shown in Table 6 and the velocity profile results are displayed in Figure 5. The crosses indicate the averaged data points to which the profiles are fitted. In the extremely stable and stable condition the velocity profiles are flat, indicating a high increase in windspeed as a function of height. The opposite is true for the unstable and extremely unstable conditions where there are small changes of velocity with height. It can also be seen that the extremely stable condition is much more prevalent a lower wind speeds. The procedure is repeated for potential temperature using Equation 5 with the corresponding stability functions from Equations 6 to 11 and the data from Table 5. This time, however, there are two unknowns, potential temperature length scale and also ground potential temperature. The resulting profiles are shown in Figure 6 . The crosses indicate the averaged data points to which the profiles are fitted. The profiles are located along the temperature axis in their expected positions with the unstable conditions occurring during the higher daytime temperatures and stable during the cooler night-time temperatures. The neutral condition appears vertical since during this condition the potential temperature gradient matches that of the dry adiabatic lapse rate. The results for potential temperature length scale and ground potential temperature are shown in Table 6. The density at the mast location is determined using moist air relations for a gas obeying the ideal gas law and the diurnally averaged pressure, relative humidity and temperature data at $5 \mathrm{~m}$ [22].

Table 6: Results from Mast 1 data analysis - Sector $180^{\circ}$

\begin{tabular}{lccccc}
\hline & $\begin{array}{c}\text { Extremely } \\
\text { Unstable }\end{array}$ & Unstable & Neutral & Stable & $\begin{array}{c}\text { Extremely } \\
\text { Stable }\end{array}$ \\
\hline$u_{*}\left[\mathrm{~m} \mathrm{~s}^{-1}\right]$ & 0.361 & 0.332 & 0.308 & 0.181 & 0.040 \\
$\theta_{*}[\mathrm{~K}]$ & -1.126 & -0.217 & 0.000 & 0.064 & 0.016 \\
$\theta\left(z_{0}\right)[\mathrm{K}]$ & 316.6 & 303.6 & 294.2 & 294.0 & 293.8 \\
$\rho\left[\mathrm{kg} \mathrm{m}^{-3}\right]$ & 1.082 & 1.082 & 1.101 & 1.097 & 1.103 \\
\hline
\end{tabular}




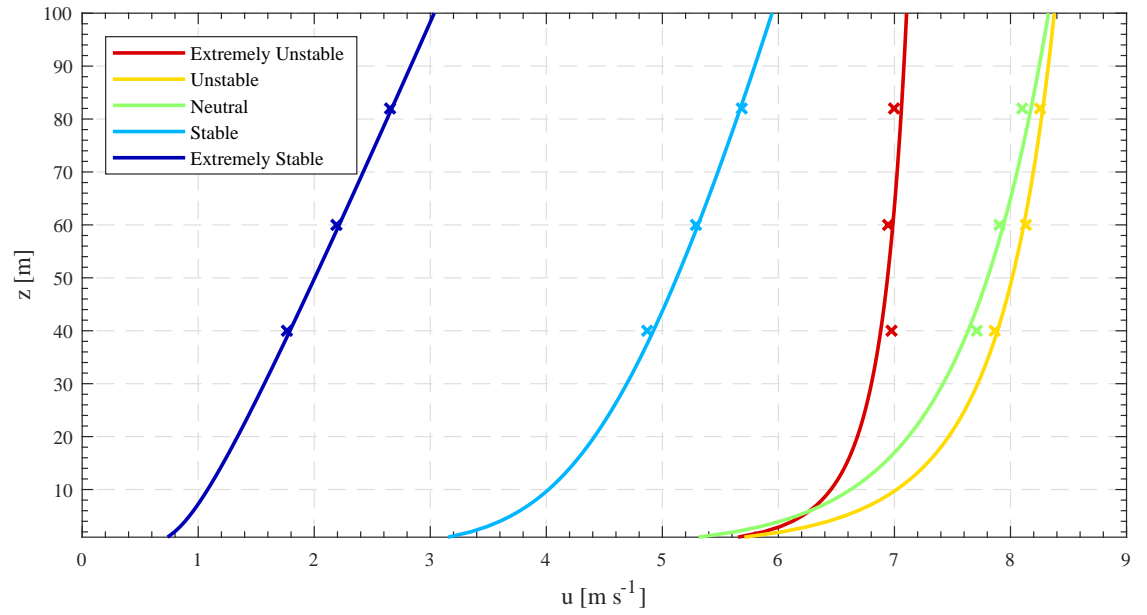

Figure 5: Measured velocity profiles - Sector $180^{\circ}$

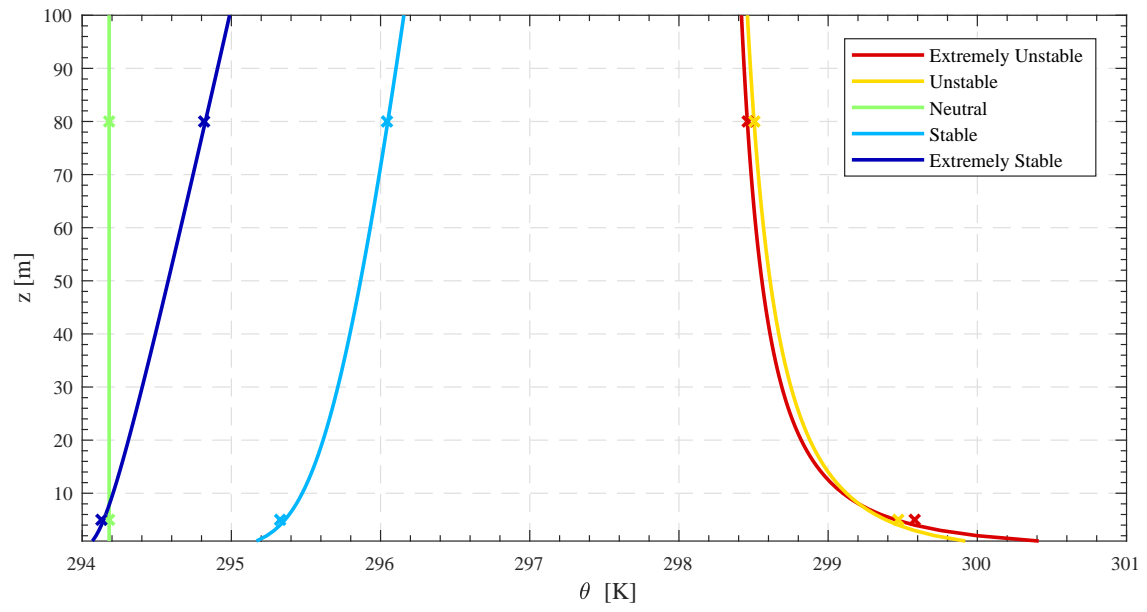

Figure 6: Measured potential temperature profiles - Sector $180^{\circ}$

The turbulence profiles for $k$ and $\epsilon$ are determined using Equation 19 and 20 with the frictional velocity in Table 6 . The resulting profiles are presented in Figures 7 and 8 . The turbulent kinetic energy $k$ has a much higher value in the unstable conditions than that of the stable regions. This is to be expected due to fluctuations present in this state. In the stable regions the fluctuations are suppressed and yield the vertical profiles with a much lower 
value than that of the other conditions. The turbulent dissipation rate $\epsilon$ profiles highlight how the dissipation is increased close to ground level.

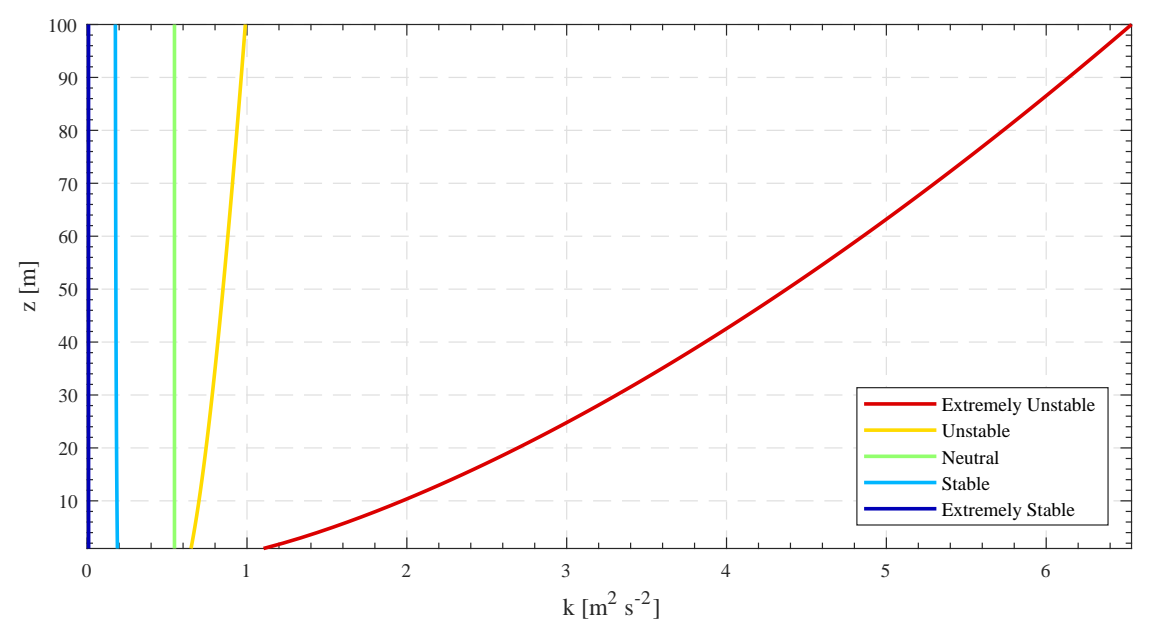

Figure 7: Turbulent kinetic energy from measurements - Sector $180^{\circ}$

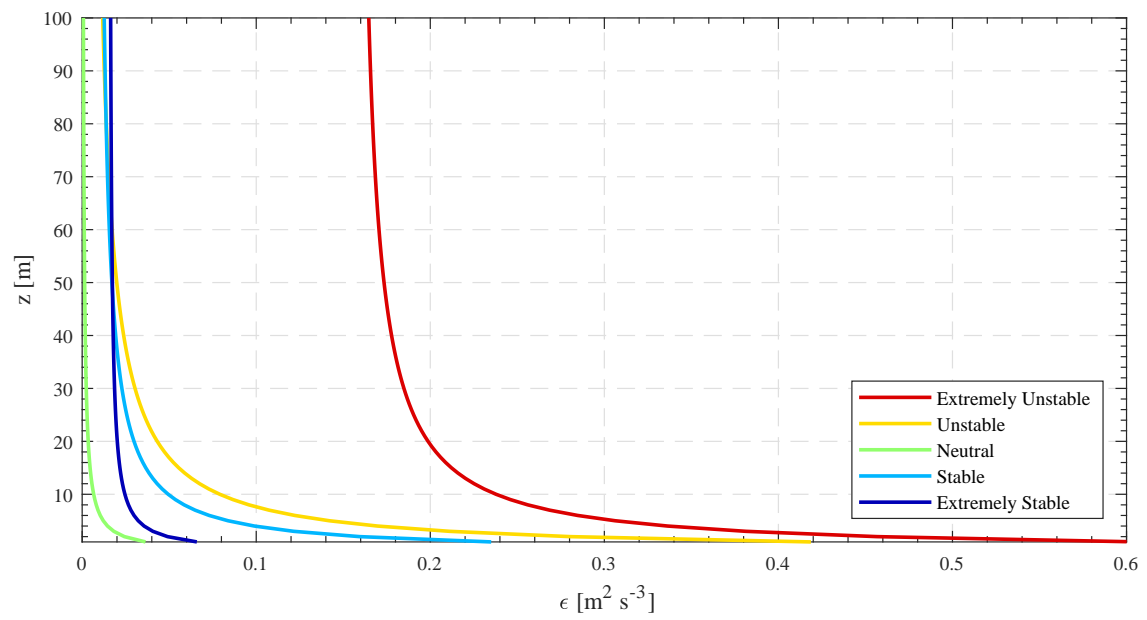

Figure 8: Turbulent dissipation rate from measurements - Sector $180^{\circ}$

\section{CFD simulations: Computational settings and parameters}

The two MOST models presented are applied to the Fluent 18.1 RANS model equations by user-defined functions (UDFs). The implementations are 
evaluated by their ability to maintain inlet profiles in an empty computational domain, the test is referred to as the horizontal homogeneity test. Thereafter they are validated using a cross prediction study using the site data described in Section 3

\subsection{User-defined functions}

The Coriolis force is included as a momentum source term and is applied in both the $\mathrm{X}$ and $\mathrm{Y}$ momentum equations in Fluent. The energy equation is not activated for the models and Fluent therefore neglects $G_{b}$ from the turbulent transport equations. For the DTU method the $S_{k}$ source term in the turbulent kinetic energy transport equation includes $S_{k M O}$ and $G_{b M O}$ and it becomes

$$
S_{k}=-\rho S_{k M O}+\mu_{t} G_{b M O}
$$

$S_{k M O}$ and $G_{b M O}$ are given by Equations 23 and 29 respectively. The velocity has two horizontal components $\left(u\right.$ and $v$ ) and $\frac{\partial U}{\partial z}$ is evaluated using the Euclidean norm shown in Equation 32.

$$
\frac{\partial U}{\partial z}=\sqrt{\left(\frac{\partial u}{\partial z}\right)^{2}+\left(\frac{\partial v}{\partial z}\right)^{2}}
$$

The frictional velocity in the $S_{k}$ source term is not kept constant but instead calculated by rewriting Equation 19 to obtain

$$
u_{*}=C_{\mu}^{1 / 4} k^{1 / 2}\left(\frac{\psi_{\epsilon}}{\psi_{m}}\right)^{-1 / 4}
$$

The $\epsilon$ source terms included in the turbulence energy dissipation rate transport equation are based on modifications to the $C_{\epsilon 3}$ constant. Fluent by default sets $C_{\epsilon 3}$ to zero. In order to reintroduce $C_{\epsilon 3}$ in a manner consistent with Equation 16, $S_{\epsilon}$ takes the following form:

$$
S_{\epsilon}=C_{\epsilon 1} \frac{\epsilon}{k} C_{\epsilon 3} G_{b}
$$

The AM is model is only valid for $-2.3<z / L<2.0$ and outside this region $S_{\epsilon}$ is set to 0 . The height of the boundary layer must be taken into account, above this height the inlet profiles and sources are set to the fixed value they would attain at the boundary layer edge. The values used in this study follow typical ABL heights. The stable boundary layer is known to be more shallow 
and is set to $600 \mathrm{~m}$ AGL, while the vertical motions in the unstable condition cause an increased boundary layer height and is set to $800 \mathrm{~m}$. The neutral boundary layer is set as $1000 \mathrm{~m}$ AGL. For the empty domain study all of the heights are, however, set to $1000 \mathrm{~m}$ AGL to not introduce any additional gradients into the solution.

\subsection{Model settings}

The inflow is along the $\mathrm{y}$ axis, the $\mathrm{x}$ axis is horizontally perpendicular to the inlet and $\mathrm{z}$ is the height above ground. The inlet boundary is an $\mathrm{x}-\mathrm{z}$ plane located upstream of the computational domain. The MOST profiles for velocity and turbulence from Equations 4, 19 and 20 are used for the AM and the DTU model inlet profiles. The neutral model uses the turbulence profiles from Equations 17 and 18. The top boundary is an $\mathrm{x}-\mathrm{y}$ plane and is also treated as an inlet using the same profiles as the inlet. The velocity is described in the y-direction only for both inlets. The sides are $y$-z planes and use symmetry boundary conditions. The outlet is a $\mathrm{x}-\mathrm{z}$ plane located downstream of the computational domain and uses an outflow condition that allows extrapolation of the relevant flow variables from inside the domain onto the outlet boundary. The bottom of the domain is set to a zero-slip wall using the method of Parente et al [23] based on the boundary conditions of Richards and Hoxey [13].

The turbulent viscosity ratio inside Fluent is based on common industrial internal flows and for the ABL simulation it is increased to $10^{10}$. The solution algorithm uses the coupled method for pressure-velocity coupling. The Presto (PREssure STaggering Option) is used for pressure spatial discretization. A least squares cell-based method is used for the gradients and all other properties adopt a second-order upwind scheme. All simulations are performed under steady-state conditions. Air is used with standard properties, except for the site simulation where the site specific air density is used. The simulations are considered converged when the residuals level out, resulting in a decrease of at least five orders of magnitude.

\subsection{Case 1: Empty domain}

The domain considered is a rectangular cuboid with dimensions of $300 \mathrm{~m}$, $10100 \mathrm{~m}$ and $1000 \mathrm{~m}$ in $\mathrm{x}, \mathrm{y}$ and $\mathrm{z}$, respectively. The domain is discretized with a uniform grid in the $\mathrm{x}$ and $\mathrm{y}$ directions of $20 \mathrm{~m}$. In the $\mathrm{z}$ direction the ground cell height equals $0.030 \mathrm{~m}$ and expands using a geometric growth ratio of 1.14 with 65 cells. The complete mesh is comprised of 492375 cells. Only 
the stability-based source terms are included in the horizontal homogeneity tests and the Coriolis force is neglected. The models were tested using the four non-neutral stability conditions, the neutral test is based on the model from Sorensen [12]. The corresponding properties for MOL and frictional velocity are listed in Table 7 . A roughness length of $0.002 \mathrm{~m}$ is used.

\subsection{Case 2: Complex terrain}

The complex terrain CFD model uses the same setup, settings and coordinate system as the empty domain model. The domain considered is a rectangular cuboid with dimensions of $35300 \mathrm{~m}, 24900 \mathrm{~m}$ and $6000 \mathrm{~m}$ in x, y and $\mathrm{z}$ directions, respectively. Using the wind farm contour data the domain is discretized via a block-structured double-O grid using the ANSYS ICEM CFD mesher. In the inner O grid the cell size is fixed to $20 \mathrm{~m}$. This covers the entire hill feature plus a $500 \mathrm{~m}$ boundary. The next block is located $3000 \mathrm{~m}$ from this boundary. In this block the cells expand in size from $20 \mathrm{~m}$ to a maximum of $50 \mathrm{~m}$ using a geometric growth ratio of 1.05. In the outer $\mathrm{O}$ grid the cells expand in size from $50 \mathrm{~m}$ to a maximum of $100 \mathrm{~m}$ using a geometric growth ratio of 1.1. The cells at the edges of the domain have a size of $100 \mathrm{~m}$. The z-direction is discretized using 80 vertical cells with a ground cell height of $0.1 \mathrm{~m}$ and a geometric growth ratio of 1.1. The complete mesh comprises of 24966291 cells. The meshing procedure and details are in accordance to generally accepted industry standards [24], the grid independence study is presented in Section 5.4. The wind farm terrain model and the mesh on the South and West faces are shown in Figure 9. The pink and black spheres respectively shows the locations of Mast 1 and 2. An artificial smoothing is applied around the terrain model so that the inlet profiles can be applied on a completely flat terrain as it removes terrain features across boundaries. 


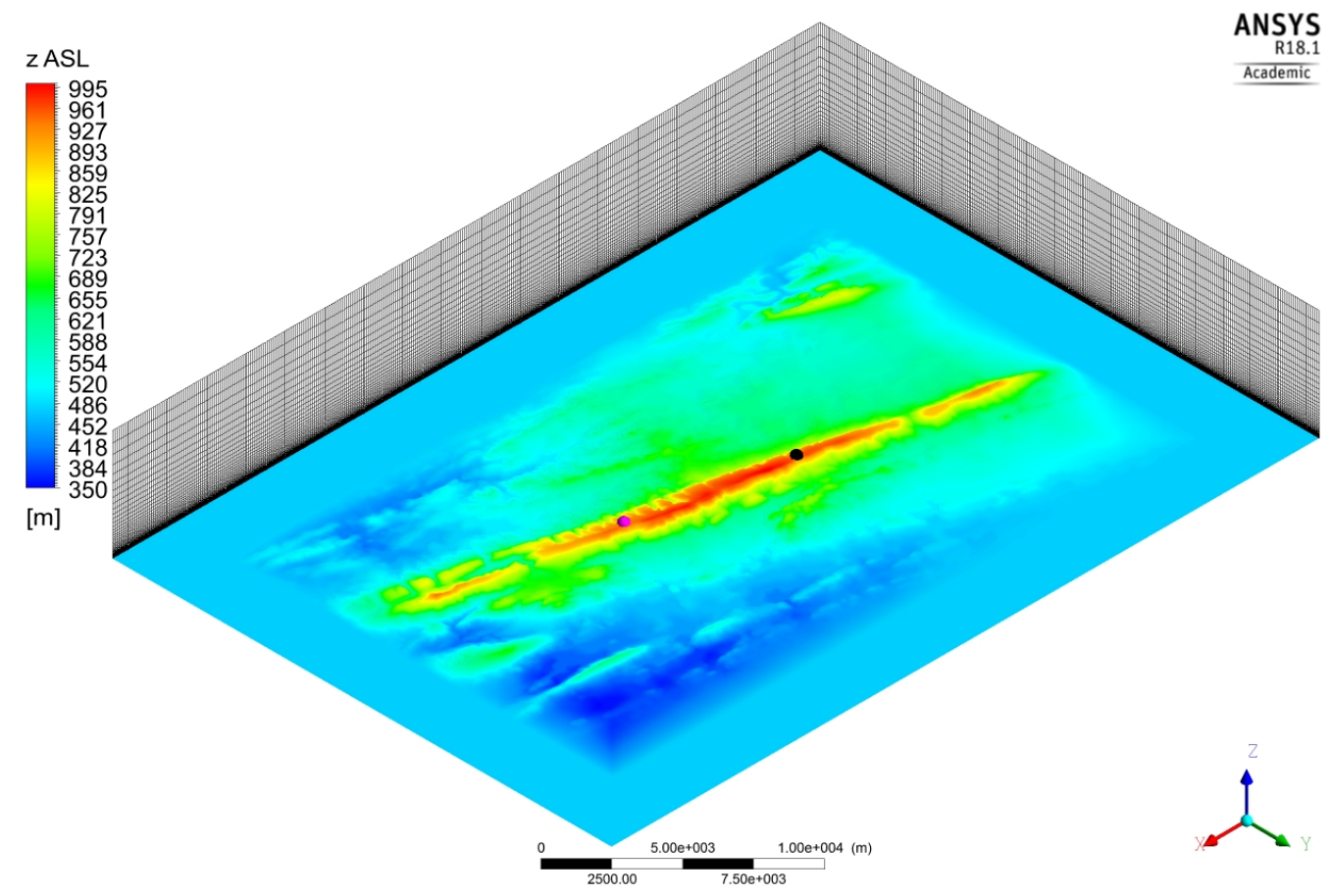

Figure 9: Wind farm terrain model coloured using height above sea level and indicating mesh density on South(x-z plane) and West faces(y-z plane)

The inlet profiles are obtained by applying the data analysis procedure to a WRF mesoscale data set obtained at the inlet location, the data are given in Table 7. The site MOL and site air density are obtained from the measured data at Mast 1 , the MOL data are given in Table 5 and the density in Table 6. A linear interpolation function is employed to determine the MOL used in the source terms. The function interpolates from the MOL obtained using the WRF data at the inlet location to the MOL calculated from the measured data at Mast 1. 
Table 7: CFD models inlet data

\begin{tabular}{cccccc}
\hline Empty Domain & $\begin{array}{c}\text { Extremely } \\
\text { Unstable }\end{array}$ & Unstable & Neutral & Stable & $\begin{array}{c}\text { Extremely } \\
\text { Stable }\end{array}$ \\
\hline$L[\mathrm{~m}]$ & -20.0 & -200.0 & N/A & 200.0 & 20.0 \\
$u_{*}\left[\mathrm{~m} \mathrm{~s}^{-1}\right]$ & 0.642 & 0.642 & 0.612 & 0.424 & 0.424 \\
\hline Complex terrain & & & & & \\
\hline$L[\mathrm{~m}]$ & -9.0 & -254.6 & $\mathrm{~N} / \mathrm{A}$ & 124.7 & 21.4 \\
$u_{*}\left[\mathrm{~m} \mathrm{~s}^{-1}\right]$ & 0.373 & 0.374 & 0.144 & 0.141 & 0.065 \\
\hline
\end{tabular}

\section{CFD simulations: Validation and results}

\subsection{Horizontal homogeneity test}

The resulting profiles at $1000 \mathrm{~m}$ and $10000 \mathrm{~m}$ downstream from the inlet for velocity, turbulent kinetic energy and dissipation are shown graphically in Figures 10 to 12. Table 8 and 9 give the absolute percentage error from the inlet profile calculated at $96.8 \mathrm{~m}$ AGL. The neutral model shows less than $1 \%$ error up to $10000 \mathrm{~m}$ for velocity with $2.44 \%$ and $4.29 \%$ for turbulent kinetic energy and turbulent dissipation rate respectively. For the non-neural model results the velocity profiles error induced at $5000 \mathrm{~m}$ is negligibly small $(<1 \%)$. At $10000 \mathrm{~m}$, however, the profiles begin to deviate more significantly in the two extreme conditions. For $k$ and $\epsilon$ the same trend is seen: Analysing the DTU $k$ error at $5000 \mathrm{~m}$ in unstable and stable conditions the error is $7.42 \%$ and $14.87 \%$ respectively. However, in the two extreme cases this error is increased in excess of $38 \%$. The AM method shows close to double the calculated errors than the DTU method in stable and unstable conditions. Comparing the turbulence profiles at $1000 \mathrm{~m}$ it is noted that both models have problems with the two extreme cases. The AM model shows difficulties in the extremely stable case with a $29.19 \%$ error. This can attributed to the fact that this model is only valid for $z / L<2.0$ and using a MOL of $20 \mathrm{~m}$, this source is only valid up to $40 \mathrm{~m}$ AGL. The $10000 \mathrm{~m}$ velocity profiles in Figure 10 highlight the issues with both models in the extreme cases: In the extremely stable condition the AM velocity is artificially increasing close to ground and in the extremely unstable conditions the DTU profile has started to decelerate. Graphically it can be seen that in extremely unstable conditions the profiles from both models lack the energy to sustain the high 
turbulence values and the profiles start to trail back compared to the inlet. In the extremely stable and stable case the AM model overshoots the $k-\epsilon$ profiles. Both methods suffer breakdowns at $10000 \mathrm{~m}$.

To match the model settings of the complex terrain model, the empty domain horizontal homogeneity test has also been performed including the Coriolis force and finite boundary layer height. The impact on the resulting profile errors were negligible.

Table 8: Percentage error at $96.8 \mathrm{~m}$ AGL - AM

\begin{tabular}{lccc}
\hline Velocity $u\left[\mathrm{~m} \mathrm{~s}^{-1}\right]$ & $1000 \mathrm{~m}$ & $5000 \mathrm{~m}$ & $10000 \mathrm{~m}$ \\
\hline Extremely Unstable & 0.12 & 0.58 & 0.30 \\
Unstable & 0.06 & 0.06 & 0.42 \\
Stable & 0.14 & 0.01 & 0.98 \\
Extremely Stable & 0.33 & 0.95 & 3.39 \\
\hline \multicolumn{1}{c}{$k\left[\mathrm{~m}^{2} \mathrm{~s}^{-2}\right]$} & $1000 \mathrm{~m}$ & $5000 \mathrm{~m}$ & $10000 \mathrm{~m}$ \\
\hline Extremely Unstable & 0.46 & 35.50 & 72.26 \\
Unstable & 1.91 & 12.76 & 34.16 \\
Stable & 10.94 & 38.92 & 60.10 \\
Extremely Stable & 29.19 & 159.21 & 520.18 \\
\hline \multicolumn{1}{c}{$\epsilon\left[\mathrm{m}^{2} \mathrm{~s}^{-3}\right]$} & $1000 \mathrm{~m}$ & $5000 \mathrm{~m}$ & $10000 \mathrm{~m}$ \\
\hline Extremely Unstable & 10.73 & 24.29 & 67.69 \\
Unstable & 1.76 & 6.22 & 21.88 \\
Stable & 15.11 & 45.09 & 57.58 \\
Extremely Stable & 41.20 & 192.82 & 591.95 \\
\hline
\end{tabular}


Table 9: Percentage error at 96.8 m AGL - DTU

\begin{tabular}{lccc}
\hline Velocity $u\left[\mathrm{~m} \mathrm{~s}^{-1}\right]$ & $1000 \mathrm{~m}$ & $5000 \mathrm{~m}$ & $10000 \mathrm{~m}$ \\
\hline Extremely Unstable & 0.03 & 0.48 & 2.83 \\
Unstable & 0.02 & 0.08 & 0.16 \\
Stable & 0.11 & 0.01 & 0.07 \\
Extremely Stable & 0.30 & 0.19 & 0.25 \\
\hline \multicolumn{1}{c}{$\quad k\left[\mathrm{~m}^{2} \mathrm{~s}^{-2}\right]$} & $1000 \mathrm{~m}$ & $5000 \mathrm{~m}$ & $10000 \mathrm{~m}$ \\
\hline Extremely Unstable & 2.50 & 47.85 & 100.00 \\
Unstable & 0.47 & 7.42 & 27.78 \\
Stable & 1.42 & 14.87 & 26.97 \\
Extremely Stable & 6.22 & 38.75 & 64.22 \\
\hline \multicolumn{1}{c}{$\epsilon\left[\mathrm{m}^{2} \mathrm{~s}^{-3}\right]$} & $1000 \mathrm{~m}$ & $5000 \mathrm{~m}$ & $10000 \mathrm{~m}$ \\
\hline Extremely Unstable & 4.37 & 35.56 & 96.74 \\
Unstable & 2.02 & 2.37 & 17.05 \\
Stable & 1.63 & 10.38 & 20.63 \\
Extremely Stable & 2.27 & 35.96 & 62.76 \\
\hline
\end{tabular}
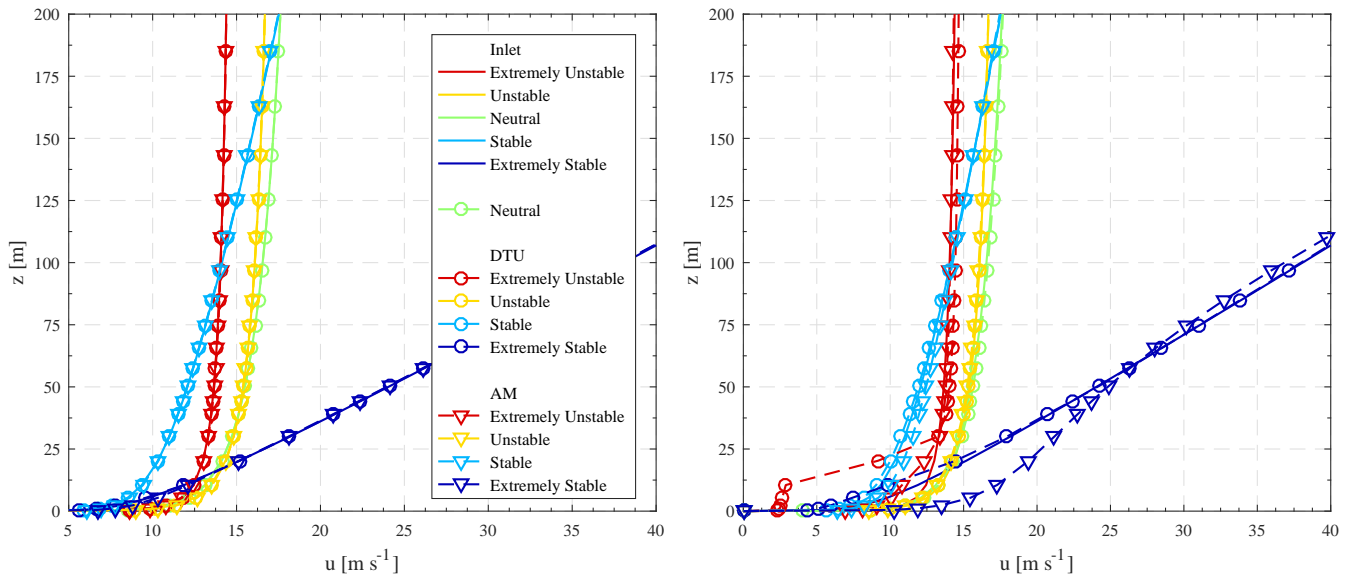

Figure 10: Velocity. Left: 1000 m Right: 10000 m 

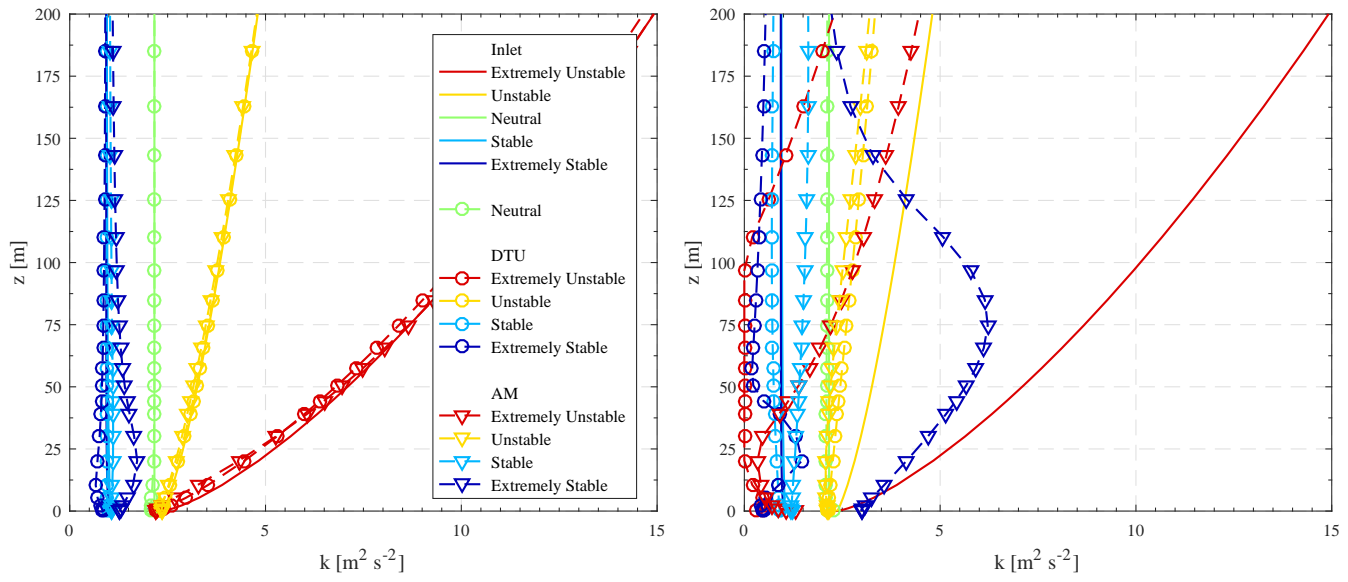

Figure 11: Turbulent kinetic energy. Left: 1000 m Right: 10000 m
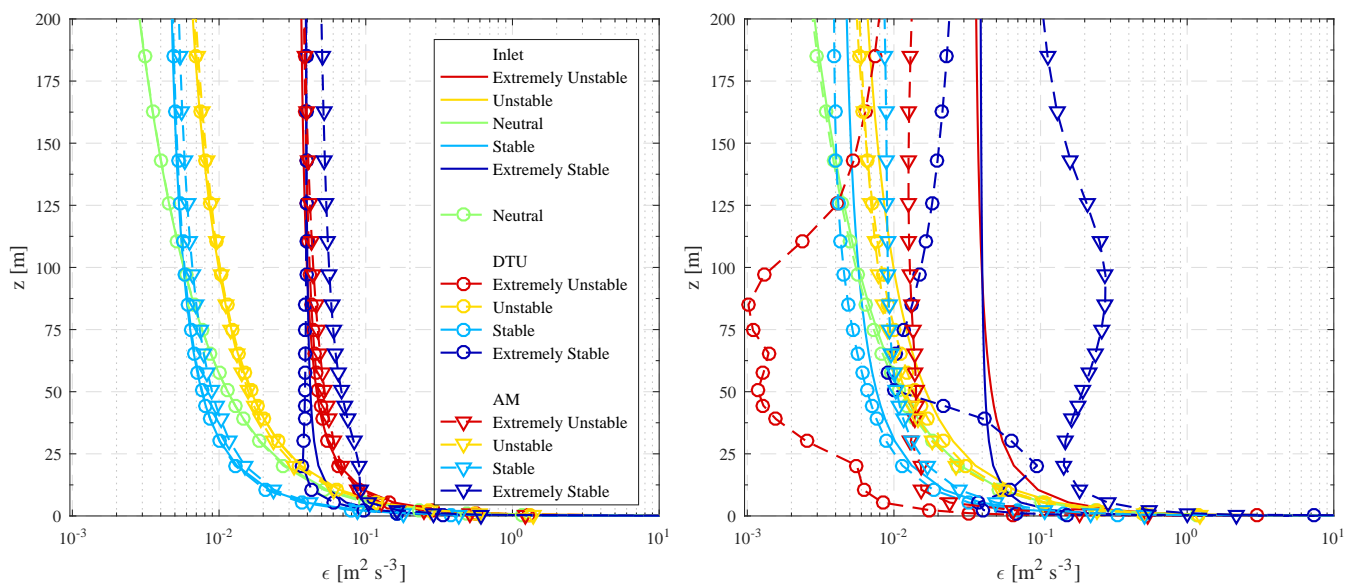

Figure 12: Turbulent dissipation rate. Left: 1000 m Right: $10000 \mathrm{~m}$

\subsection{Complex terrain model validation}

Both ABL CFD models along with the stability and site data are used to test and validate the models in a complex terrain. Using the CFD results three transfer functions are created from the velocity magnitude at $40 \mathrm{~m}$, $60 \mathrm{~m}$ and $82 \mathrm{~m}$ AGL at both mast locations. These heights correspond to the measurement heights of the masts. The velocity transfer function $\Gamma$ is defined as

$$
\Gamma=\frac{u_{M 2 \mathrm{CFD}}}{u_{M 1 \mathrm{CFD}}}
$$


where M1 and M2 denote Mast 1 and 2. Using the transfer function it is the possible to obtain the predicted velocity at Mast 2 using Equation 36.

$$
u_{\text {Pred. }}=\Gamma u_{M 1 \mathrm{CFD}}
$$

The percentage cross-prediction error is then calculated using Equation 37

$$
\text { Error }=100 \times \frac{\left|u_{M 2 \text { Meas. }}-u_{\text {Pred. }}\right|}{u_{\text {Meas. }}}
$$

The prediction results at $82 \mathrm{~m}$ AGL for both models are given in Table 10 . The measured vs. predicted velocity profiles are shown in Figure 13. The measured velocity is obtained by applying the data analysis procedure to the data measured at mast 2 . The crosses indicate the mean measured velocity from Mast 2 and the solid line is the velocity profile fit for these points. The circles and triangles are the predicted velocities using Equation 36.

\begin{tabular}{|c|c|c|c|c|c|}
\hline & $\begin{array}{c}\text { Extremely } \\
\text { Unstable }\end{array}$ & Unstable & Neutral & Stable & $\begin{array}{c}\text { Extremely } \\
\text { Stable }\end{array}$ \\
\hline$u_{\text {Meas. }}\left[\mathrm{m} \mathrm{s}^{-1}\right]$ & 7.33 & 7.69 & 6.85 & 5.88 & 2.78 \\
\hline$u_{\text {Pred. }}$ DTU $\left[\mathrm{m} \mathrm{s}^{-1}\right]$ & 7.34 & 8.92 & & 5.32 & 2.53 \\
\hline$u_{\text {Pred. }} \mathrm{AM}\left[\mathrm{m} \mathrm{s}^{-1}\right]$ & 7.28 & 9.09 & & 5.49 & 2.24 \\
\hline Error DTU [\%] & 0.08 & 15.97 & & 9.49 & 9.10 \\
\hline Error AM [\%] & 0.74 & 18.17 & & 6.74 & 19.36 \\
\hline
\end{tabular}

Table 10: Mast 2 cross prediction results at $82 \mathrm{~m}$

${ }^{1}$ Using the neutral model - $u_{\text {Pred. Neutral }\left[\mathrm{m} \mathrm{s}^{-1}\right]}$

${ }^{2}$ Using the neutral model - Error Neutral [\%]

The cross-prediction results show that both models were able to accurately capture the two main stability conditions onsite. The model results give an error of less than $1 \%$ in the extremely unstable condition, this condition is present onsite $36 \%$ of the time. The most dominating condition is the stable condition which is present $40 \%$ of the time. In this condition both models have errors of less than $10 \%$. In the extremely stable condition at $82 \mathrm{~m}$ the DTU model outperformed the AM model by $10 \%$. The profiles in Figure 13 illustrate this as one of the shortcomings of the AM model, which is only valid for $z / L<2$ and using the mast MOL of $21.4 \mathrm{~m}$ this model results in a loss of validity for heights greater than $42.8 \mathrm{~m}$. This can be seen 
in the profiles by noting the small error at $40 \mathrm{~m}$ extremely stable compared to the increased error it exhibits at $82 \mathrm{~m}$.

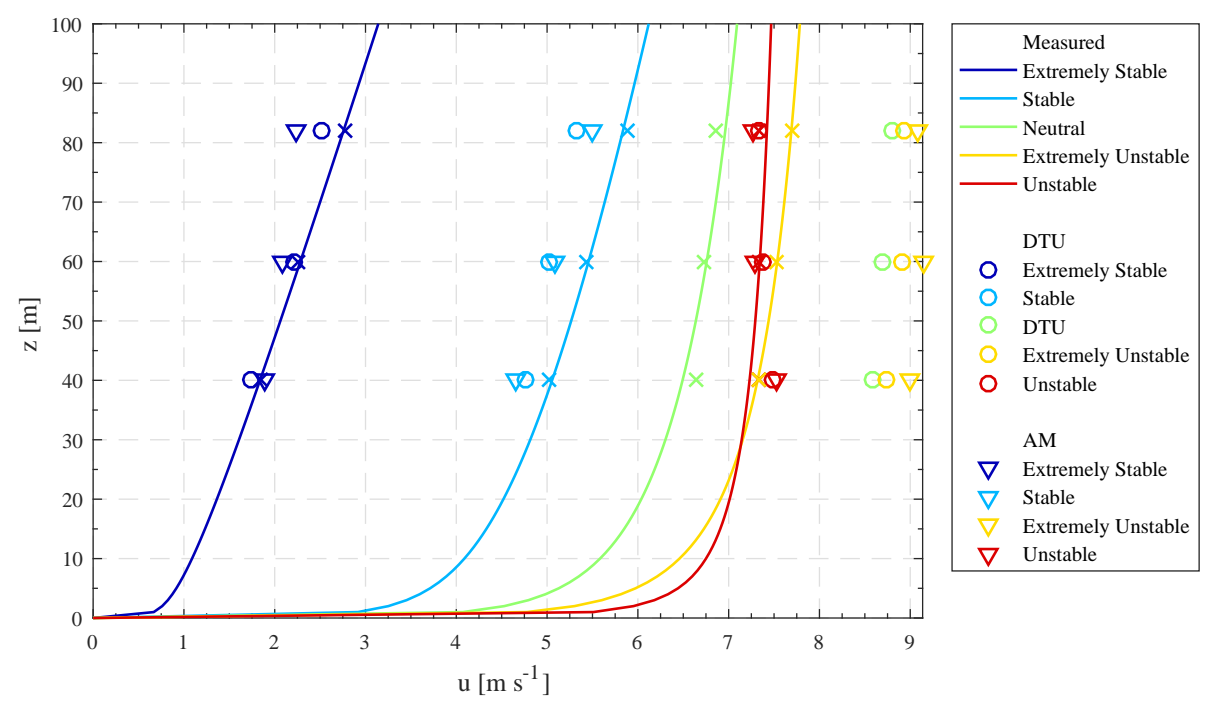

Figure 13: Predicted vs. measured wind speed profiles at Mast 2

Marginally increased errors are present for both models in the unstable condition. The worst performing model is the neutral model with a $28.5 \%$ error. This high error can be attributed to the increased variance in the neutral data. The neutral condition is only present for $11 \%$ of the measurement campaign and by analysing the diurnal stability classification in Figure 3 the neutral condition does not have a fixed period in which it occurs, instead occurring at any time of day. There is thus higher variance in the neutral data which causes the increased error. In order to understand the total error a frequency weighted error is calculated. This error is weighted according to the stability frequency classification and is determined as

$$
\text { Total Error }=\frac{\sum_{j=1}^{5} \text { frequency }_{j} \times \text { Error }_{j}}{\sum_{j=1}^{5} \text { frequency }_{j}}
$$

where $j$ indicates the five stability classes. The error is obtained from Table 10 and the frequency is the stability frequency classification from the measured data in Table 4. The total error is calculated as $8.55 \%$ for the DTU model and $8.54 \%$ for the AM model. There is thus negligible difference between these two models in the total cross prediction error and both 
models have an error of less then $10 \%$ in cross prediction. From the profiles in Figure 13 it can be seen that both models were able to accurately predict the shape of the wind profiles. In stable conditions the high shear exponent causes the more flattened profiles while for unstable conditions the profiles are closer to upright as there is very little change in velocity with height. The only condition that has an error in this regard is the extremely unstable condition in which both models have problems predicting the complete vertical profile, instead over-predicting the velocity at $42 \mathrm{~m}$.

Using the CFD results and taking the inverse tangential of the $\mathrm{z}$ and $\mathrm{y}$ velocity using Equation 39 it is possible to calculate the inflow angle experienced at the mast measurement points.

$$
\Omega=\tan ^{-1}\left(\frac{u_{3}}{u_{2}}\right)
$$

Table 11 shows the inflow angle obtained from the CFD results at $40 \mathrm{~m}$ above ground level Mast 1. The largest inflow angle is less than $10^{\circ}$ for which the CLASSCUP study showed anemometers are able to accurately capture the change in inflow.

Table 11: Inflow angle in degrees from CFD results at $40 \mathrm{~m}$ above ground level

\begin{tabular}{lcc|cc}
\hline & \multicolumn{2}{c|}{ AM } & \multicolumn{2}{c}{ DTU } \\
\hline & Mast 1 & Mast 2 & Mast 1 & Mast 2 \\
Extremely Stable & 2.97 & 2.01 & 3.25 & 9.67 \\
Extremely Unstable & 2.62 & 4.94 & 2.03 & 1.62 \\
Stable & 2.41 & 3.50 & 2.59 & 3.18 \\
Unstable & 2.72 & 2.84 & 2.71 & 3.08 \\
Neutral & $2.24^{1}$ & $2.38^{1}$ & N/A & N/A \\
\hline
\end{tabular}

${ }^{1}$ Using the neutral model

\subsection{Stability lifting/blocking effects}

Non-neutral stratification can also cause lifting/blocking effects when the windfields encounter a terrain feature like a hill [1] [16]. In neutral conditions the wind profiles would go smoothly over the hill, in stable conditions they 
are more likely to flow around the hill rather than over. This is due to the buoyancy effects in the stable condition that counteract lifting. In unstable conditions the profile rises over the hill and is more prone to continue to rise downstream of the hill due the buoyancy effect caused by the displaced profiles which are warmer than the surrounding air [16].

This effect is present in the CFD results. In Figure 14 the neutral velocity streamlines over a specific hill section in the terrain are shown. The hill has a slight opening towards the Eastern part. The streamlines are released directly in front and perpendicular to the hill. In the neutral condition the streamlines flowing over the hill are completely straight and smooth with no turbulent mixing behind the hill. In Figures 15 and 16 the performance of the DTU model in unstable and stable conditions is shown. Both models exhibited the exact same behaviour and were accurately able to capture the lifting and blocking effects.

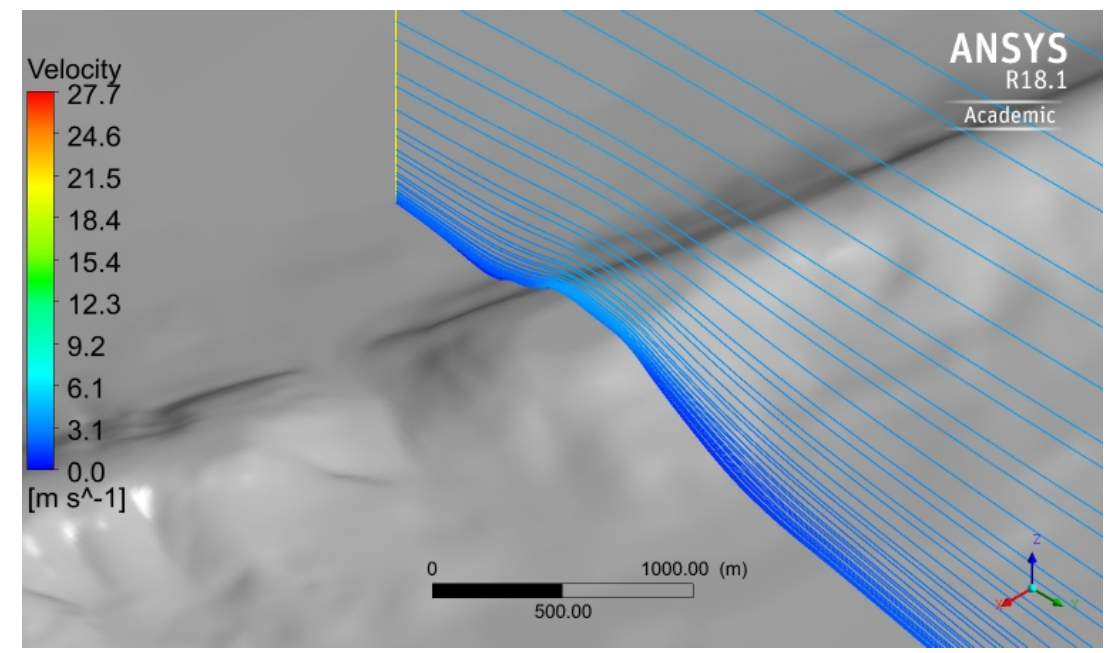

Figure 14: Velocity streamlines over terrain feature under neutral stratification

In stable conditions the streamlines flow around the hill towards the opening instead of over. This effect causes the high wind shear values experienced in stable conditions. The streamlines close to ground flow around instead of up the hill. A slow moving parcel of air is thus experienced close to ground on top of the hill, the streamlines higher above ground do flow over the hill and where these two meet there is an increased change of velocity with height which leads to the high wind shear values. In unstable conditions the streamlines go over the hill and travel onwards downstream of the hill instead of 
flowing smoothly down. This causes a turbulent mixing zone that is present behind the hill, this zone was captured by both models. This increased turbulence is the reason why in unstable conditions the turbulence intensity is increased from the neutral and stable conditions.

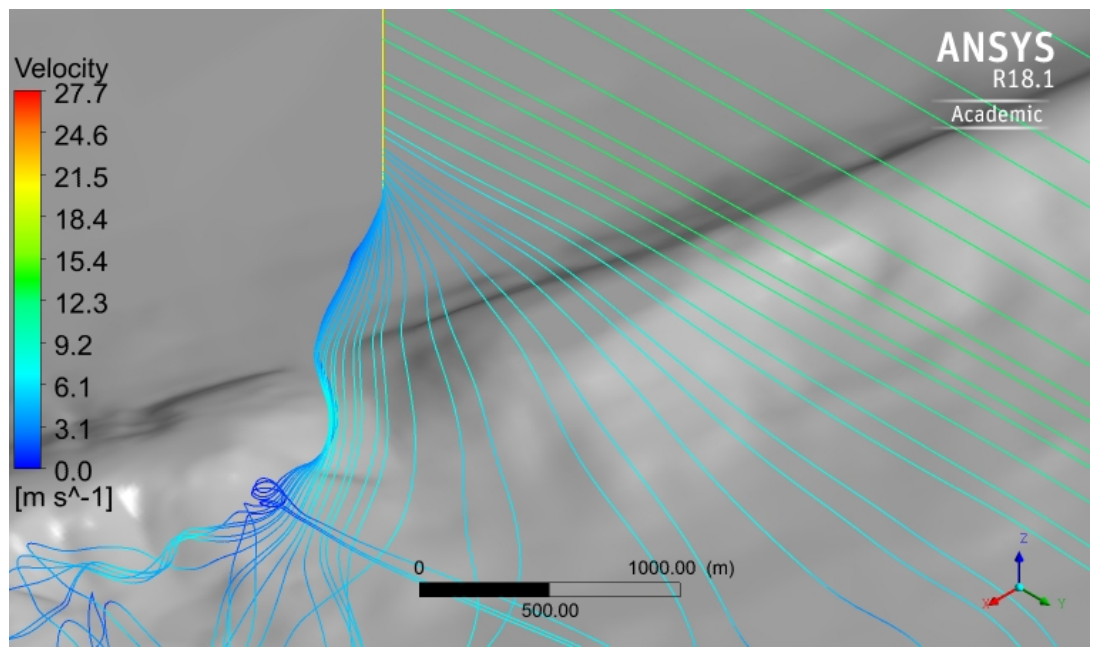

Figure 15: Effect of atmospheric stability on velocity streamlines - DTU model Stable

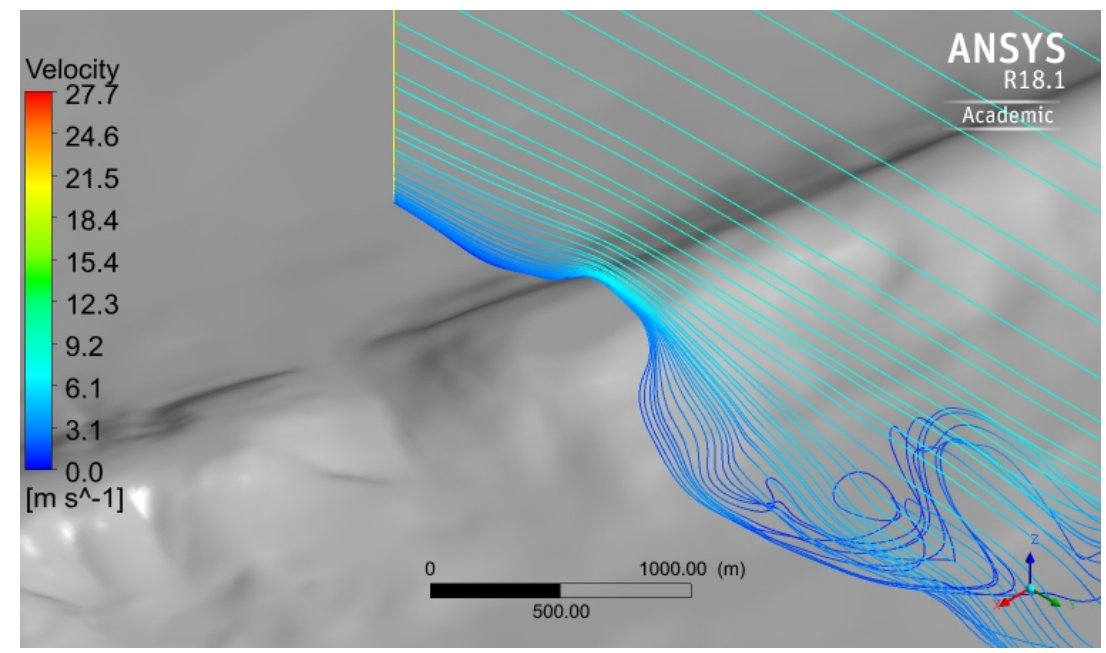

Figure 16: Effect of atmospheric stability on velocity streamlines - DTU model Unstable 


\subsection{Grid independence study}

To ensure that the complex terrain model CFD results are not influenced by the mesh used, a grid independence study was performed. The $20 \mathrm{~m}$ central resolution (24966291 cells) was set to $10 \mathrm{~m}$ and $30 \mathrm{~m}$, resulting in cell counts of 51762799 and 15028923 respectively. The fine resolution roughly doubles the number of cells in the simulation. The change of CFD model wind speed at mast 1 with number of cells is shown in Figure 17. Using the $20 \mathrm{~m}$ resolution as reference, the variation for $10 \mathrm{~m}$ and $30 \mathrm{~m}$ is $1.3 \%$ and $2.1 \%$ respectively. These small levels of variation are well within the overall wind farm model uncertainty and matches well with the results from the DTU Perdigo CFD Grid Study [24]. It can be concluded that the mesh used in the complex terrain model produces grid independent results.

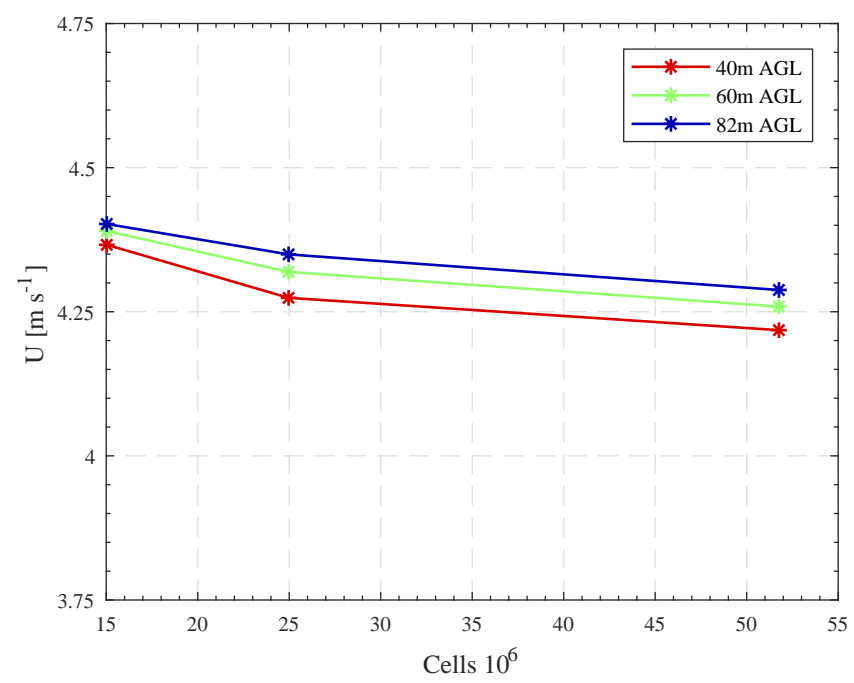

Figure 17: Effect of atmospheric stability on velocity streamlines - DTU model Unstable

\section{Discussion}

From the horizontal homogeneity test it can be concluded that care should be taken in the extreme cases for both models. The models are generally presented in literature under standard non-neutral conditions, omitting the extreme conditions. However, based on an unpublished study, the DTU model author was able to accurately model the extreme cases using the EllipSys3D CFD code. The errors reported could be due the Fluent code's solver and 
wall functions, the author is currently in the process of testing the stability models in other CFD codes to understand if the errors are code specific. The DTU model shows less error due to the fact that the model is analytically in balance for all values of $z / L$, including both extreme cases. Under standard non-neutral conditions both models perform well with the DTU model outperforming the AM model. However, both models have trouble sustaining profiles over large distances. ABL CFD models are known to be problematic in flat terrain [25]. For this reason care should be taken to not use excessively long upstream inlet distances, both models showed suitable profiles up to $5000 \mathrm{~m}$. From the results it can be concluded that the models can account for atmospheric stability and that horizontal homogeneity of the profiles is obtained.

The complex terrain results showed that both models were able to successfully model the onsite effects of atmospheric stability. Applying the developed data analysis procedure on a WRF mesoscale data point at the inlet and the primary mast at the centre of the site yielded accurate inputs to the CFD model. The cross-prediction study successfully validated the ABL model with low errors experienced in all non-neutral conditions. A total error of $8.5 \%$ was obtained for both models. The greatest errors occurred for conditions which are non-dominant and it can be concluded that care should be taken when analysing these conditions due the naturally increased variance in non-dominating conditions. The lifting and blocking effects known to be caused by stratification were also found to be in accordance to those described in literature. The difference in errors from both models are negligible and not one clear model performed better than the other. The only major difference in cross-prediction error is in the extremely stable condition, however, as this condition is not one of the dominating conditions using it to decide on one model or the other is premature. Further cross prediction studies on wind farm locations with other conditions and terrains are therefore required to accurately comment on which model is best. Both models are successfully validated for modelling atmospheric stability.

\section{Conclusion}

This study presented an atmospheric boundary layer CFD model which aims to describe neutral and non-neutral wind flow over complex terrain using site-specific stability parameters. The model was successfully validated using 
a horizontal homogeneity test and a cross-prediction study from a proposed wind farm location.

The prevalence and effect of atmospheric stability on the windfields were determined by applying Monin-Obukhov Similarity Theory (MOST) to two years of onsite measured time series data. The results indicated strong nonneutral conditions with neutral conditions present for only $11 \%$ of the measurement period. The data analysis method which applies MOST to measured time series data and uses the diurnally weighted average to determine sector-wise mean conditions and profiles were developed by the authors. It is to the best of the authors' knowledge a novel implementation of MOST to determine atmospheric stability and vertical profiles of velocity, temperature and turbulence.

Two MOST models were tested, the results from both models in the horizontal homogeneity test highlighted problems modelling extreme conditions and maintaining profiles for extended distances. Both models were able to accurately maintain profiles of velocity and turbulence up to $5000 \mathrm{~m}$. In the complex wind farm terrain study both MOST models were evaluated using the CFD results to cross predict stability-dependent velocity profiles. During the two main stability conditions experienced, both models gave errors of less than $10 \%$. The DTU model showed it is more capable of dealing with the extreme cases than the AM model. Using the frequency classification, both models gave a total error of $8.5 \%$ which proves both models were successfully able to accurately model non-neutral flows onsite. To the best of the authors' knowledge this study presents the first application of the DTU model to complex terrain as well as the first comparison of the AM and DTU models for a complex terrain.

The advantage of using the proposed ABL CFD model is the ability to model more of the large-scale physical mechanisms of the ABL. This allows greater accuracy in the design of wind farms. Using this method, the measured stability-dependent profiles can be accurately extrapolated to any proposed turbine location onsite. In summary, the results showed that the implemented MOST modifications and developed methods are applicable and reproduced the main wind flow characteristics in non-neutral flows over complex wind farm terrains. 


\section{Acknowledgements}

The authors' would like to thank the University of Pretoria for the support throughout the project. Computational resources were provided by the CHPC (Centre for High Performance Computing). Financial support and commercial data were provided by Siemens Gamesa Renewable Energy.

\section{References}

[1] M. P. van der Laan, M. C. Kelly, N. N. Sørensen, A new k-epsilon model consistent with MoninObukhov similarity theory, Wind Energy 20 (3) (2017) 479-489. doi:10.1002/we.2017.

[2] M. Motta, R. Barthelmie, P. Vlund, The influence of non-logarithmic wind speed profiles on potential power output at danish offshore sites, Wind Energy 8 (2005) 219-236. doi:10.1002/we.146.

[3] C. Alinot, C. Masson, Aerodynamic Simulations of Wind Turbines Operating in Atmospheric Boundary Layer With Various Thermal Stratifications, ASME 2002 Wind Energy Symposium (July) (2002) 206-215. doi:10.1115/WIND2002-42.

[4] J. M. Wallace, H. P. V., Atmospheric Science An Introductory Survey, Vol. 2, Elsevier, 2006.

[5] H. A. Panofsky, J. A. J. A. Dutton, Atmospheric turbulence : models and methods for engineering applications, John Wiley, New York, 1984.

[6] A. Sathe, J. Mann, T. Barlas, W. A. A. M. Bierbooms, G. J. W. Van Bussel, Influence of atmospheric stability on wind turbine loads, Wind Energy 16 (7) (2013) 1013-1032. doi:10.1002/we.1528.

[7] T. Koblitz, CFD Modeling of Non-Neutral Atmospheric Boundary Layer Conditions, Ph.D. thesis, Danmarks Tekniske Universitet (2013).

[8] A. J. Dyer, A review of flux-profile relationships, Boundary-Layer Meteorology 7 (3) (1974) 363-372. doi:10.1007/BF00240838.

[9] J. S. Irwin, F. S. Binkowski, Estimation of the monin-obukhov scaling length using on-site instrumentation, Atmospheric Environment 15 (6) (1981) 1091 - 1094. doi:https://doi.org/10.1016/0004-6981(81)90111-6. 
[10] ANSYS, ANSYS Fluent Theory Guide 18.1, Tech. rep., Ansys Fluent $18.1(2017)$.

[11] H. Versteeg, W. Malaasekera, An introduction to computational fluid dynamics: the finite volume method, 2nd Edition, Pearson Education Limited, 1995.

[12] N. Sorensen, General purpose flow solver applied to flow over hillsPublished 2003.

[13] P. J. Richards, R. P. Hoxey, Appropriate boundary conditions for computational wind engineering models using the k-e turbulence model, Journal of Wind Engineering and Industrial Aerodynamics 46 (1993) 145-153. doi:http://dx.doi.org/10.1016/0167-6105(93)90124-7.

[14] A. Sogachev, M. Kelly, M. Y. Leclerc, Consistent Two-Equation Closure Modelling for Atmospheric Research: Buoyancy and Vegetation Implementations, Boundary-Layer Meteorology 145 (2) (2012) 307-327. doi:10.1007/s10546-012-9726-5.

[15] C. Alinot, C. Masson, k-epsilon Model for the atmospheric boundary layer under various thermal stratifications, Journal of Solar Energy Engineering-Transactions of The ASME 127 (4) (2005) 438-443. doi:10.1115/1.2035704.

[16] C. Meissner, A. R. Gravdahl, B. Steensen, Including thermal effects in CFD simulations, Journal of the environmental sciences (2009) 5.

[17] J. F. Manwell, J. G. McGowan, A. L. Rogers, Wind Energy Explained, 2nd Edition, Wiley, 2009. doi:10.1002/9781119994367.

[18] T. G. Farr, P. A. Rosen, E. Caro, R. Crippen, R. Duren, S. Hensley, M. Kobrick, M. Paller, E. Rodriguez, L. Roth, D. Seal, S. Shaffer, J. Shimada, J. Umland, M. Werner, M. Oskin, D. Burbank, D. E. Alsdorf, The shuttle radar topography mission, Reviews of Geophysics 45 (2) (2007) RG2004. doi:10.1029/2005RG000183.

[19] M. Strack, MEASNET Procedure Evaluation of Site-Specific Wind Conditions Released, DEWI Magazin 36 (2010) 76-81. 
[20] J.-Å. Dahlberg, T. F. Pedersen, P. Busche, ACCUWIND - Methods for Classification of Cup Anemometers, Tech. Rep. May 2006 (2006).

[21] EMD, EMD International A/S EMD-WRF South Africa Mesoscale Data, [Online] (Date last accessed 2017-10-28) http://www.emd.dk/windpro/mesoscale-data/emd-wrf-south-africamesoscale-data/.

[22] R. S. Davis, Equation for the Determination of the Density of Moist Air, Metrologia 29 (1) (1992) 67.

[23] A. Parente, C. Gorlé, J. van Beeck, C. Benocci, Improved k- $\epsilon$ model and wall function formulation for the RANS simulation of ABL flows, Journal of Wind Engineering and Industrial Aerodynamics 99 (4) (2011) 267-278. doi:10.1016/j.jweia.2010.12.017.

[24] A. Bechmann, Perdigo CFD Grid Study, DTU Wind Energy, Denmark, 2016.

[25] B. Blocken, T. Stathopoulos, J. Carmeliet, CFD simulation of the atmospheric boundary layer: wall function problems, Atmospheric Environment 41 (2) (2007) 238-252. doi:10.1016/j.atmosenv.2006.08.019. 